\title{
An Exploration of Heterogeneity in Supernova Type la Samples
}

\author{
Ujjaini Alam ${ }^{a, b}$ Jeremie Lasue ${ }^{b}$ \\ ${ }^{a}$ Physics \& Applied Mathematics Unit, Indian Statistical Institute, Kolkata India \\ ${ }^{b}$ IRAP, Université de Toulouse, CNRS, UPS, CNES, Toulouse, France \\ E-mail: ujjaini.alam@gmail.com, jeremie.lasue@irap.omp.eu
}

\begin{abstract}
We examine three SNe Type Ia datasets: Union2.1, JLA and Panstarrs to check their consistency using cosmology blind statistical analyses as well as cosmological parameter fitting. We find that the Panstarrs dataset is the most stable of the three to changes in the data, although it does not, at the moment, go to high enough redshifts to tightly constrain the equation of state of dark energy, $w$. The Union2.1, drawn from several different sources, appears to be somewhat susceptible to changes within the dataset. The JLA reconstructs well for a smaller number of cosmological parameters. At higher degrees of freedom, the dependence of its errors on redshift can lead to varying results between subsets. Panstarrs is inconsistent with the other two datasets at about $2 \sigma$ confidence level, and JLA and Union2.1 are about $1 \sigma$ away from each other. For the $\Omega_{0 m}-w$ cosmological reconstruction, with no additional data, the $1 \sigma$ range of values in $w$ for selected subsets of each dataset is two times larger for JLA and Union2.1 as compared to Panstarrs. The range in $\Omega_{0 m}$ for the same subsets remains approximately similar for all three datasets. We find that although there are differences in the fitting and correction techniques used in the different samples, the most important criterion is the selection of the SNe, a slightly different SNe selection can lead to noticeably different results both in the purely statistical analysis and in cosmological reconstruction. We note that a single, high quality low redshift sample could help decrease the uncertainties in the result. We also note that lack of homogeneity in the magnitude errors may bias the results and should either be modeled, or its effect neutralized by using other, complementary datasets. A supernova sample with high quality data at both high and low redshifts, constructed from a few surveys to avoid heterogeneity in the sample, and with homogeneous errors, would result in a more robust cosmological reconstruction.
\end{abstract}




\section{Contents}

1 Introduction $\quad 1$

2 Supernova Data 2

3 Statistical analysis of Supernovae data $\quad 3$

$\begin{array}{lll}3.1 & \text { Exploratory data analysis } & 5\end{array}$

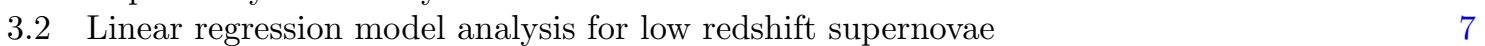

$\begin{array}{lll}3.2 .1 & \text { Methodology } & 8\end{array}$

$\begin{array}{llr}3.2 .2 & \text { Results } & 8 \\ \end{array}$

\begin{tabular}{ll}
3.3 & Nonlinear regression model analysis for all supernovae data \\
\hline
\end{tabular}

$\begin{array}{lll}3.3 .1 & \text { Methodology } & 15\end{array}$

$\begin{array}{lll}3.3 .2 & \text { Results } & 15\end{array}$

4 Cosmology $r 2$

\begin{tabular}{ll}
4.1 & Methodology \\
\hline & 22
\end{tabular}

$\begin{array}{llr}4.2 & \text { Results } & 23\end{array}$

5 Conclusions $r$

6 Acknowledgements 29

\section{Introduction}

Observations of distant type Ia supernovae by the Supernova Cosmology Project (SCP) and the High Redshift Search Team (HZT) [1, 2], in the late 1990s, uncovered one of the most mystifying cosmological phenomena today- the accelerated expansion of the Universe. One way to explain this observational result is to theorize the existence of a new form of energy, with negative pressure, often called "dark energy". Another is to postulate a modification of the rules of gravity. Many different models have been suggested for these, some of which are reviewed and analyzed in [3-19]. Current cosmological observations are commensurate with the cosmological constant [20,21], where the dark energy equation of state is -1 and its energy density is constant. However, other dark energy models are by no means ruled out [22], and the search for the true nature of dark energy is a continuing process.

Different observations have provided indirect evidence for the presence of dark energy, such as the Cosmic Microwave Background Radiation (CMB) [20], and Baryon Acoustic Oscillations (BAO) [23-25]. Nevertheless, the most direct evidence for the accelerated expansion of the Universe remains the type Ia SNe. These observations are not without their share of controversy. Type Ia SNe are considered to be "calibrated candles", i.e., although their peak brightness is not identical to each other, after being subjected to a stretch factor, they fall on the same light-curve with remarkable uniformity. The evidence for this is purely empirical and although the SNe type Ia as a group follow the stretch relations extremely well, there remain some outliers. There is no strong theoretical proof yet as to why the type Ia SNe should be "calibrated candles", nor as to why there should not be any magnitude evolution between near and distant SNe. Indeed, it is possible to study potential magnitude evolution in type Ia SNe to examine whether sufficient magnitude evolution could negate or weaken the result of accelerated expansion of the universe. Some groups have studied the techniques for correcting for stretch and color in SNe [26]. Others have studied the possibility of non Gaussian distribution of $\mathrm{SNe}$ [27] or the effect of cosmic dust on SNe magnitudes [28]. The impact of metallicity on the SNe rates has been examined in [29]. Host selection for Type Ia SNe has also been studied recently [30]. The effect of interstellar dust on the luminosity of distant SNe, the exact mechanism behind type Ia SNe explosions, all these are as yet ill understood. As such, results from the type Ia SNe have met 
with some scepticism from the community. Despite the many unknowns, the SNe results are however strongly corroborated by many other observations, and over the years, as the SNe data have grown qualitatively and quantitatively, the proof for accelerated expansion of the universe has only grown stronger.

Currently, the focus is on constraining dark energy parameters such as the dark energy equation of state today, $w_{0}$, and its evolution with the scale factor, $w_{a}$. While SNe cosmology is entering its precision era, the apparent heterogeneity in the SNe Ia available to us remains a cause for concern. There have been attempts to quantify the effects of this heterogeneity on the cosmological reconstruction $[31,32]$, as yet there are no conclusive results. In this work we study the three most used and comprehensive currently available SNe Ia datasets to understand the consistency within and across the different samples and to assess how this may affect the cosmological analysis. The paper is arranged as follows: section 2 contains a description of the SNe data used, section 3 describes the results obtained by applying cosmology-independent statistical techniques to SNe Ia data, in section 4 we obtain constraints on cosmological parameters, and in section 5 we present our conclusions.

\section{Supernova Data}

The first type Ia SNe datasets hinting at the accelerated expansion of the universe were published in the late $1990 \mathrm{~s}$ by $[1,2]$. At this time, only $36 \mathrm{SNe}$ were available from SCP, and 42 from HZT with fairly high magnitude errors. Naturally these few observations could not constrain dark energy parameters to appreciable levels. Since then, various SNe teams have augmented the type Ia SNe dataset and also utilized improved techniques for data reduction. In 2003 the HZT team published the luminosity-redshift relation of 230 type Ia SNe [33]. This paper constrained the equation of state of dark energy at present to $-1.48<w<-0.72$ at $2 \sigma$ for a flat $w \mathrm{CDM}$ model. In the same year, [34] added a further 23 high redshift SNe to this set, doubling the high redshift $\mathrm{SNe}$ sample at $z>0.7$. In 2004, [35] added 16 SNe from the Hubble Space Telescope (HST) to the previous samples, and defined a subset of $157 \mathrm{SNe}$ with well defined spectroscopic features, host redshift, well-sampled colour and light curves as the Gold dataset. With added priors on $\Omega_{0 m}$ from WMAP and the Two-Degree Field Galaxy Redshift Survey (2dfGRS), this constrained the equation of state of dark energy at present to $w=-1.08_{-0.18}^{+0.20}$. All these analyses used the MLCS2k2 light-curve fitter. The first results from the SuperNova Legacy Survey (SNLS) were reported in 2005 [36] with 71 new high redshift SNe, leading to $\Omega_{0 m}=0.271 \pm 0.021$ (stat) \pm 0.007 (syst), $w=-1.023 \pm 0.090$ (stat) \pm 0.054 (syst) with added constraints from BAO and assumed flatness. This group introduced the SALT-I fitter for light-curves. The 2007 results from the ESSENCE survey [37] were commensurate with the SNLS results, with $w=-1.05_{-0.12}^{+0.13}$ and $\Omega_{0 m}=0.274_{-0.020}^{+0.033}$ in a similar analysis. The 2008 Union2 dataset [38] comprised of 307 SNe from ESSENCE, SNLS, HST and older datasets, using the SALT-I fitter. These, with BAO and CMB observations, led to constraints of $w=-0.969_{-0.063}^{+0.059}(\text { stat })_{-0.066}^{+0.063}$ (sys). In 2009, magnitude-redshift relations for 185 type Ia SNe observed at the F. L. Whipple Observatory of the Harvard-Smithsonian Center for Astrophysics (CfA) were reported in [39]. This resulted in the creation of the Constitution dataset, which added the Union2 dataset to the CfA3 data and BAO to report $w=-1.013_{-0.068}^{+0.066}$ (stat) \pm 0.11 (syst). This paper also carried out a comparison of the various light-curve fitting methods: MLCS2k2, SALT-I and SALT-II.

The first season of the Sloan Digital Sky Survey-II (SDSS-II) Supernova Survey filled the "redshift desert" between low and high redshift SNe [40], and combining these with the ESSENCE, SNLS and HST SNe, obtained $\Omega_{0 m}=0.307 \pm 0.019$ (stat) \pm 0.023 (syst), $w=-0.76 \pm 0.07$ (stat) \pm 0.11 (syst) for the MLSC2k2 fitter, and $\Omega_{0 m}=0.265 \pm 0.016$ (stat) \pm 0.025 (syst), $w=-0.96 \pm 0.06$ (stat) \pm 0.12 (syst) for the SALT-II fitter. This paper highlighted the interesting fact that different empirical light curve fitters could give significantly different cosmological results for the same SNe. A further 20 high redshift SNe from the HST Cluster Supernova Survey added to the existing Union2 collection, and analyzed using the SALT-II fitter, forms the Union2.1 set with 580 SNe. This results in constraints of $w=-1.013_{-0.073}^{+0.068}$ [41]. The three year SNLS program along with other previous SNe yielded a total of 472 high quality SNe (with $242 \mathrm{SNe}$ from SNLS alone), resulting in constraints of $w=-0.91_{-0.24}^{+0.17}($ stat + sys $), \Omega_{0 m}=0.18 \pm 0.10($ stat + sys $)$ [42]. The $146 \mathrm{SNe}$ at redshifts 
$0.03<z<0.65$ discovered during the first 1.5 years of the Pan-STARRS1 Medium Deep Survey [43], added to existing low redshift data, as well as BAO, CMB and $H_{0}$ data, provide a constraint of $\Omega_{0 m}=0.280_{-0.012}^{+0.013}, w=-1.166_{-0.069}^{+0.072}$. A joint analysis of SNe obtained by the SDSS-II and SNLS collaborations using the SALT-II fitter, totals 740 spectroscopically confirmed type Ia supernovae with high quality light curves. This "JLA" sample yields $w=-1.027 \pm 0.055$ (stat + sys) with added $\mathrm{BAO}$ and CMB constraints [44].

Several points are to be noted about these SNe surveys:

- Firstly, the SNe data has gone from less than 100 SNe with errors $\sigma_{\mu} \sim 1-10[1,2]$ to $\gtrsim 500$ SNe with errors $\sigma_{\mu} \sim 0.1-1.0$. These observations have seen more than an order of magnitude improvement both in numbers and accuracy.

- Many different light curve fitters are now available for analysis, e.g., MLCS2K2 [45]; Stretch [46]; SALT-I, SALT-II [47]; SiFTO [48]; BayeSN [49, 50]. These have improved the accuracy of individual SNe light curves to $\sim 5 \%$, but there remain inconsistencies between the different fitters, and cosmological results may vary depending on the fitter used. Currently, the most commonly used fitter is SALT-II.

- Although many different surveys have reported high redshift type Ia SNe, the low redshift range $z \lesssim 0.1$ does not have many new observations. This range, however, is crucial for the normalization of the data. Thus, even the new and high quality high redshift data have to use the older low redshift data, which is a fairly heterogeneous dataset.

- Supernova data, by itself, can only constrain the total energy density. Thus, in order to extract information on dark energy from it, it is imperative to include information on the curvature of the Universe (e.g., from CMB) and the matter density (e.g., from BAO). Even with these information, it may not be possible to obtain reasonable constraints on both the equation of state at present and its evolution over time. Thus, it is often the practice to quote results for a constant equation of state dark energy. This is not theoretically a very viable dark energy model. The data should therefore reach an accuracy where we are able to constrain both the present value and rate of evolution of the dark energy equation of state. This could be achieved by better quality SNe data, augmenting SNe data with other, complementary observations, or by using more sophisticated statistical analysis techniques.

In this work we assess the consistency of the SNe databases and the effect any inconsistencies may have on the cosmological parameters constrained from them.

\section{Statistical analysis of Supernovae data}

The SALT-II model transforms the light-curve fit parameters into distances as:

$$
\mu=m_{B}+\alpha \cdot X-\beta \cdot C-M_{B}
$$

where $\mu$ is the distance modulus which contains the cosmological information, $m_{B}$ is the peak B-band brightness, $X$ is a light-curve shape parameter, and $C$ is a colour parameter. $\alpha$ is determined by the relation between luminosity and stretch while $\beta$ is determined by the relation between luminosity and color. $M_{B}$ is the absolute B-band magnitude of a fiducial SNe Ia with $X=0, C=0$. The parameters $\alpha, \beta, M_{B}$ are nuisance parameters that are fitted simultaneously with the cosmological parameters. Both the absolute magnitude $M_{B}$ and $\beta$ parameter depend on host galaxy properties. However, the effect of this on cosmological results is expected to be negligible, so we neglect this dependence for our analysis as this is a sub-dominant systematic effect [51].

We have at our disposal at present, three main SNe type Ia datasets which have been processed using the SALT-II model. These have some common SNe, and some SNe which are survey specific.

- The Union2.1 dataset [41]: This dataset contains 580 SNe gathered from 19 different sources. The break-up of the data in samples is shown in Table 1. As we see, there are 19 different samples from which the data is taken, these include SNe from the early 1990s up to 2011. 
Table 1. Sample distribution of Union2.1 data

\begin{tabular}{|cccccccc|}
\hline Sample & No. & Redshift & $\sigma_{m_{B}}$ & $\sigma_{X}$ & $\sigma_{C}$ & $\sigma_{\mu_{B}}$ & Reference \\
\hline 1 & 18 & $0.02-0.10$ & $0.04-0.19$ & $0.10-0.93$ & $0.01-0.09$ & $0.16-0.22$ & {$[53]$} \\
2 & 6 & $0.02-0.03$ & $0.07-0.14$ & $0.12-0.21$ & $0.01-0.03$ & $0.09-0.15$ & {$[54]$} \\
3 & 11 & $0.02-0.12$ & $0.03-0.14$ & $0.14-0.49$ & $0.01-0.04$ & $0.16-0.22$ & {$[55]$} \\
4 & 15 & $0.02-0.05$ & $0.05-0.18$ & $0.14-1.54$ & $0.01-0.05$ & $0.22-0.31$ & {$[56]$} \\
5 & 8 & $0.02-0.16$ & $0.03-0.15$ & $0.10-0.85$ & $0.01-0.04$ & $0.08-0.16$ & {$[38]$} \\
6 & 94 & $0.02-0.08$ & $0.04-0.21$ & $0.08-1.30$ & $0.01-0.11$ & $0.16-0.29$ & {$[39]$} \\
7 & 18 & $0.02-0.08$ & $0.03-0.15$ & $0.05-0.31$ & $0.01-0.04$ & $0.10-0.18$ & {$[57]$} \\
8 & 129 & $0.04-0.42$ & $0.02-0.11$ & $0.12-1.56$ & $0.01-0.09$ & $0.11-0.25$ & {$[58]$} \\
9 & 11 & $0.24-0.97$ & $0.05-0.15$ & $0.30-1.85$ & $0.04-0.27$ & $0.31-0.81$ & {$[59]$} \\
10 & 33 & $0.17-0.83$ & $0.05-0.27$ & $0.15-1.67$ & $0.05-0.43$ & $0.42-1.01$ & {$[2]$} \\
11 & 19 & $0.34-0.98$ & $0.07-0.26$ & $0.30-2.88$ & $0.04-0.37$ & $0.20-0.73$ & {$[34]$} \\
12 & 5 & $0.18-0.27$ & $0.03-0.12$ & $0.19-0.51$ & $0.02-0.09$ & $0.19-0.26$ & {$[60]$} \\
13 & 11 & $0.36-0.86$ & $0.05-0.09$ & $0.25-0.53$ & $0.03-0.08$ & $0.10-0.20$ & {$[61]$} \\
14 & 72 & $0.25-1.01$ & $0.03-0.14$ & $0.13-1.01$ & $0.01-0.14$ & $0.13-0.43$ & {$[36]$} \\
15 & 74 & $0.16-0.78$ & $0.05-0.21$ & $0.20-1.85$ & $0.04-0.19$ & $0.20-0.39$ & {$[62]$} \\
16 & 6 & $0.28-1.06$ & $0.06-0.12$ & $0.39-1.01$ & $0.04-0.18$ & $0.18-0.46$ & {$[33]$} \\
17 & 30 & $0.22-1.39$ & $0.07-0.21$ & $0.21-1.23$ & $0.03-0.13$ & $0.20-0.37$ & {$[63]$} \\
18 & 6 & $0.51-1.12$ & $0.06-0.15$ & $0.26-0.85$ & $0.03-0.36$ & $0.09-0.95$ & {$[64]$} \\
19 & 14 & $0.62-1.41$ & $0.09-0.17$ & $0.31-1.53$ & $0.05-0.20$ & $0.17-0.56$ & {$[41]$} \\
\hline
\end{tabular}

Samples 1-7 contain low redshift data, with error bars for the distance modulus ranging between $0.08<\sigma_{\mu}<0.31$ (and that for the peak B-band brightness between $0.02<\sigma_{m_{B}}<0.21$ ). These SNe cannot constrain the cosmology very stringently since at low redshifts the universe follows the simple linear Hubble law, but these are essential to provide a low- $z$ anchor to the Hubble diagram. For the high redshift data in samples $8-19$, we have $0.09<\sigma_{\mu}<1.08$ and $0.03<\sigma_{m_{B}}<0.31$. The highest redshift data is in sample 16 at $z=1.4$, the largest samples are sample 6 for low redshift, sample 8 for low to mid redshifts, and samples 14, 15 for high redshift data. These samples might be expected to dominate the results.

- The JLA dataset [44]: This dataset combines the SNLS and SDSS SNe to create an extended sample of 740 SNe along with existing lower redshift SNe. The sample distribution of the data is shown in Table 2. The two biggest samples are: the SDSS data at low to mid redshift, with peak B-band brightness errors between $0.11<\sigma_{m_{B}}<0.17$; and the SNLS three year data at high redshift (up to $z=1.06$ ) with $0.02<\sigma_{m_{B}}<0.21$. The remaining data is from older samples. Various checks have been carried out for consistency, bias and systematic uncertainties on this dataset and it is expected to be a more homogeneous dataset than the Union2.1 data.

- The Panstarrs dataset [43]: This dataset contains 311 SNe, with the high redshift SNe measured from the Pan-STARRS1 Medium Deep Survey, and the low redshift SNe from various sources. The sample distribution is shown in Table 3. The Panstarrs data only goes up to $z \leq 0.634$, but its high redshift data is the most homogeneous, observed from a single survey. A few low redshift SNe were also observed by this survey, but they are too few in number to make a difference, and low redshift SNe from other surveys are required for normalization. In the low redshift data, JRK07 is a composite of the samples 1-4 of the Union2.1 sample. The cosmological results so far obtained from this dataset are markedly different from the results from the other two samples. This may however be an effect of less data points and lower redshifts.

We note here that some data subsets, although given different names by different groups, represent subgroups of the same observational sets, and many of these are common to the 3 SNe samples. 
Table 2. Sample distribution of JLA data

\begin{tabular}{|ccccccc|}
\hline Sample & No. & Redshift & $\sigma_{m_{B}}$ & $\sigma_{X}$ & $\sigma_{C}$ & Reference \\
\hline Calan/Tololo & 17 & $0.01-0.08$ & $0.14-0.16$ & $0.07-0.39$ & $0.03-0.06$ & {$[53]$} \\
CfAI & 7 & $0.02-0.05$ & $0.14-0.16$ & $0.12-0.27$ & 0.03 & {$[55]$} \\
CfAII & 15 & $0.01-0.06$ & $0.14-0.18$ & $0.04-0.35$ & $0.02-0.04$ & {$[56]$} \\
CfAIII & 55 & $0.01-0.07$ & $0.14-0.17$ & $0.03-0.47$ & $0.02-0.04$ & {$[39]$} \\
CSP & 13 & $0.01-0.08$ & $0.14-0.17$ & $0.02-0.11$ & 0.02 & {$[57]$} \\
Other & 11 & $0.01-0.08$ & $0.14-0.17$ & $0.04-0.23$ & $0.02-0.04$ & {$[38,54]$} \\
SDSS & 374 & $0.04-0.40$ & $0.11-0.17$ & $0.03-1.38$ & $0.01 s-0.09$ & {$[65]$} \\
SNLS & 239 & $0.13-1.06$ & $0.09-0.14$ & $0.05-1.64$ & $0.02-0.09$ & {$[42]$} \\
HST & 9 & $0.84-1.29$ & $0.11-0.13$ & $0.29-0.69$ & $0.04-0.11$ & {$[63]$} \\
\hline
\end{tabular}

Table 3. Sample distribution of Panstarrs data

\begin{tabular}{|cccccccc|}
\hline Sample & No. & Redshift & $\sigma_{m_{B}}$ & $\sigma_{X}$ & $\sigma_{C}$ & $\sigma_{\mu_{B}}$ & Reference \\
\hline JRK07 & 48 & $0.02-0.12$ & $0.03-0.24$ & $0.04-2.87$ & $0.03-0.07$ & $0.15-0.41$ & {$[45,53,55,56]$} \\
CfA3 & 84 & $0.01-0.08$ & $0.03-0.19$ & $0.06-1.08$ & $0.03-0.07$ & $0.14-0.31$ & {$[39]$} \\
CfA4 & 43 & $0.01-0.07$ & $0.03-0.26$ & $0.10-0.98$ & $0.03-0.11$ & $0.19-0.48$ & {$[66]$} \\
CSP & 22 & $0.01-0.08$ & $0.02-0.17$ & $0.04-0.35$ & $0.02-0.04$ & $0.13-0.25$ & {$[57]$} \\
Panstarrs-1 & 113 & $0.03-0.63$ & $0.03-0.23$ & $0.12-1.98$ & $0.02-0.22$ & $0.10-0.64$ & {$[43]$} \\
\hline
\end{tabular}

Examples of subsets which are drawn from the same set of observations are: Sample 1 in Union2.1 and the Calan-Tololo sample in JLA, Sample 3 of Union2.1 and CfA2 of JLA, Sample 4 of Union2.1 and CfA2 of JLA, Sample 6 of Union2.1 and CfA3 in both JLA and Panstarrs, Samples 1-4 of Union2.1 and JRK07 in Panstarrs, Sample 7 of Union2.1 and CSP in JLA and Panstarrs, Sample 16 of Union2.1 and HST of JLA and Panstarrs. Most of the low redshift data is drawn from the same observations while the high redshift data is mostly different in the three datasets.

An additional effect to be considered is that of intrinsic dispersion. This quantity can be estimated in different ways. For the Panstarrs data, the intrinsic scatter attributed to color variation and that attributed to luminosity variation lead to a fairly large variation in the parameter $\beta$, in the Union2.1 analysis, the median $\sigma_{\text {sample }}$ is used as a measure of intrinsic scatter, while JLA uses color corrected intrinsic dispersion. Neither of the latter two analyses reports a large variation in $\beta$. The accurate measure of intrinsic dispersion continues to be a matter of some debate, and the study of the variation of standardisation parameters such as $\beta$ with intrinsic dispersion remains inconclusive at present. Since the main motivation of this paper is to compare the different datasets for statistical stability, rather than to extract cosmological information, hence we avoid using the very different values of $\sigma_{i n t}$ used in the different samples. We rather use the simplest method and use the median $\sigma_{\text {sample }}$ to correct for $\sigma_{\text {int }}$. Utilizing a bias correction with different simulations of data using different values of $\beta$, as in [43], may result in as much as $4 \%$ difference in the distance moduli, and may change cosmological results, but we constrain ourselves to the simplest form of intrinsic scatter to reduce potential uncertainities pertaining to different systematics in the different datasets.

In the rest of this section, we use the free source $\mathrm{R}$ software package to develop the data exploration [67].

\subsection{Exploratory data analysis}

A first look at the data consistency may be obtained by exploratory data analysis using multivariate projections of the different parameters fitted for each $\mathrm{SNe}\left(m_{B}, \sigma_{m_{B}}, X, \sigma_{X}, C, \sigma_{C}\right)$. This is especially useful to characterize clustering and similarity within datasets and get insight into their variations and consistency [68, 69]. 
The data matrix is normalized by centering and scaling the columns by their mean and variance. Then principal components analysis is performed to determine the directions of maximum variance of the data. This is equivalent to projecting the data along the first two eigenvectors of the data matrix [70]. The cumulative variance on the principal components follows approximately the same distribution for every dataset, which indicates a similar spread of the data in the multivariate space for Panstarrs, JLA and Union2.1. The cumulative variance of the first two principal components correspond to about $60 \%$ of the total variance for every set. This low value indicates a relatively even spread of the data points in the different dimensions of the multivariate space, or equivalently that no dimensional direction singly dominates the extent of the set of data points, as expected from uncorrelated variables.

The projection on the first two principal components for each dataset is plotted in Figure 1, with the contribution of the different loadings represented by the length and direction of the arrows, with labels corresponding to the relevant parameters. One notes that the projection of Panstarrs and Union2.1 datasets are consistent with each other. Their parameter loadings appear distributed over a half-space in a similar manner, from the direction of the arrows projected in the plane to the same parameters being projected in the same directions. As is expected, the $m_{B}$ loading (corresponding also to the variation in redshift) separates the low redshift data (JRK07, CFA3, CFA4, CSP) from the high redshift data (PS1) of the Panstarrs dataset. This is also the case for the Union2.1 dataset, for which the $m_{B}$ direction indicates the transition from the circles (low redshift) to the triangles (high redshift). The other loadings do not appear to play a significant role in separating the data, and no other obvious clustering appears from the projection on the first two principal components for Panstarrs or Union2.1, indicating consistency in the spread of data. One can notice though that in the Union2.1 dataset, subset 8 appears to form a tighter cluster of data points in this projection, due to lower errors of the fitting parameters as compared to the other subsets, which can also be noticed from Table 1.

The JLA projection presents some interesting structure and clustering, which appears correlated with the data subsets. Here, one notices that as compared to Panstarrs or Union2.1, the loading directions and modules are different, showing a different contribution of the various parameters to the principal components. Firstly, unlike in the other datasets, one notices that both $m_{B}$ and $\sigma_{m_{B}}$ separate the low- $z$ (SNLS, HST, SDSS, CT, CFA1, Others) data points from the medium- $z$ (CFA3) and high- $z$ (CFA2, CSP) data points. Secondly, in this dataset the clusters are more strongly separated than for the other datasets owing to the influence of $\sigma_{m_{B}}$. Furthermore, additional clustering is visible in the low redshift cluster where CFA1 and CT appear to separate from the main cluster of data points at low- $z$ due to the influence of $\sigma_{X}$ and $\sigma_{C}$. Overall, the effect of clustering due to different errors for the fitting parameters should not play a significant role in the retrieved cosmology model parameters as these effects are rather small. But the fact that they correlate with separate subsets of the JLA dataset indicates specific differences due to the origin of the data and incomplete consistency of the datasets.

In statistics, when the variability of an observation changes systematically as a function of a predictor variable, then the dataset is said to be heteroscedastic [71]. In this case, the usual assumption that the observations have a constant variance accross the response range is not satisfied and should be taken into account in the analysis. In figure 2, the plot of $\sigma_{m_{B}}$ - the fitted errors of the magnitude $m_{B}$ - as a function of $\ln (z)$ for the three datasets shows different variabilities in the errors as a function of the redshift. The Panstarrs dataset appears to be fairly well behaved, with a relatively constant distribution of the errors and few outliers. The error behaviour of Union2.1 is dependent on the subset considered with the lowest errors for subset 8 . One notices the heteroscedasticity of the data with larger errors at low and high redshifts. This would indicate that $\mathrm{SNe}$ with extreme redshifts may present higher chances of being outliers to the magnitude-redshift relationship. Finally, the JLA $\sigma_{m_{B}}$ present a strong dependence on the subset considered with clear clustering of the data at low, mid and high redshifts, combined with an apparent heteroscedasticity of the data at low and high redshifts. It is interesting to note the increase in errors at the low and high redshifts, which may translate into increased uncertainties for the SNe located at the extremes of the surveys. In such cases, care needs to be taken to make sure that the data points at low and high redshifts do not strongly bias the 


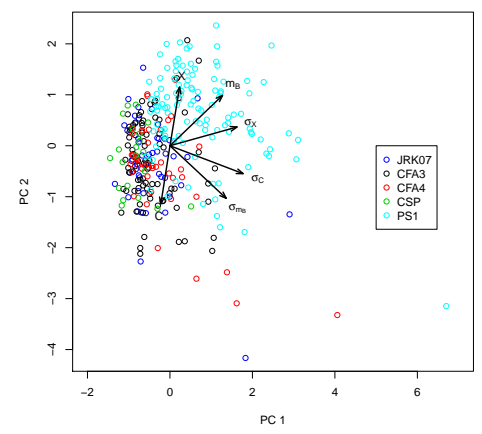

(a)

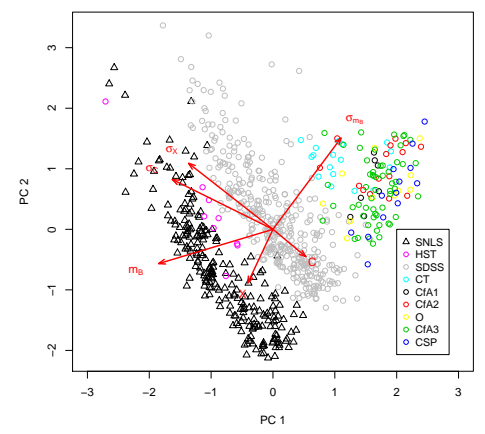

(b)

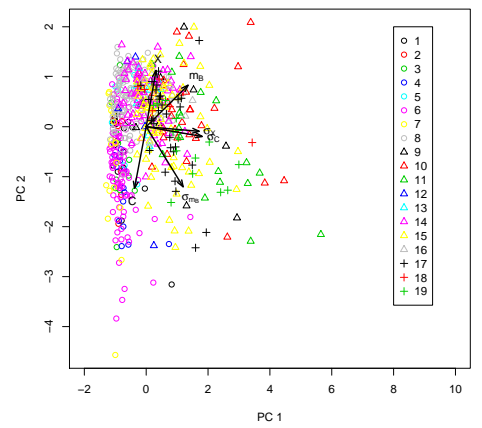

(c)

Figure 1. First 2 principal components projections of the parameter matrices for (a) Panstarrs, (b) JLA and (c) Union2.1 datasets. The scores of the data points along the principal components are used to generate the map, and the loadings of the different directions to the eigenvectors are represented by the arrows, with the label indicating which parameter direction is used. The colours and data point shapes correspond to the different subsets of each dataset as explained in the legend of the figures.

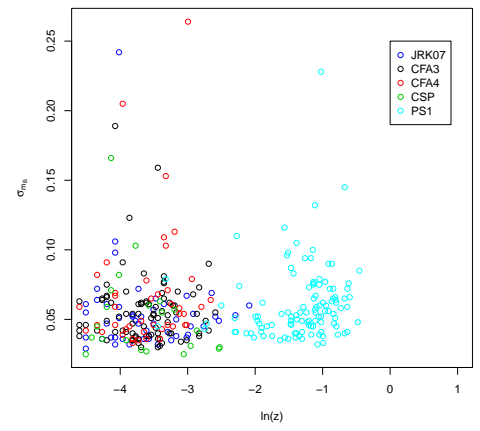

(a)

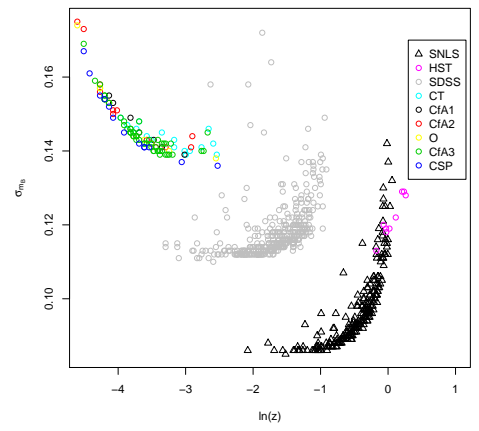

(b)

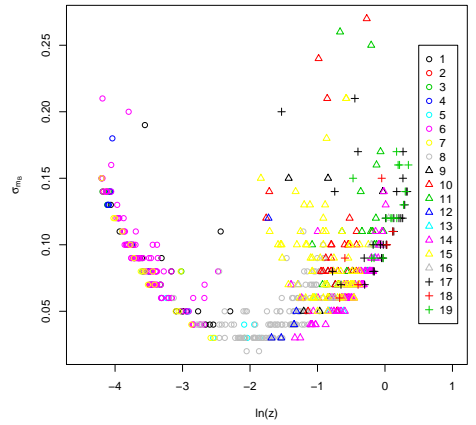

(c)

Figure 2. Residuals for the fitted magnitude, $\sigma_{m_{B}}$, as a function of $\ln (z)$ for (a) Panstarrs, (b) JLA and (c) Union2.1 datasets. Differences in the behaviour of the residuals for the fitted magnitude in each dataset are apparent. The colours and data point shapes correspond to the different subsets of each dataset as explained in the legend of the figures.

parameter estimations for the magnitude-redshift fit [71].

Further multidimensional scaling analyses do not indicate that more clustering of the data is present in any dataset. In the next sections, we focus our analysis on the relationship between the magnitude and redshift, which is used to retrieve the cosmological model parameters.

\subsection{Linear regression model analysis for low redshift supernovae}

We first consider the low redshift $\mathrm{SNe}$, since in these, $\ln (z)$ has a linear, cosmology-independent relationship to $\mu$, and therefore to $m_{B}$. According to the Hubble law, the recession velocity of the system is given as $v_{r}=H d$. In terms of redshift, $v_{r}=c z$. Thus for nearby SNe, we may approximate the distance to $d_{L}=c z / H_{0}$ where $H_{0}=$ Hubble parameter at present. Thus the magnitude is related to redshift as:

$$
\mu=5 \log _{10} d_{L}+25=5 \log _{10} z+\left(5 \log _{10} \frac{c}{H_{0}}+25\right),
$$


Table 4. Optimization parameters for the linear regression for the different datasets at low $z(z \leq 0.1)$.

\begin{tabular}{|l|l|ccc|}
\hline Data Subset & Dataset & Intercept & Slope & $R^{2}$ \\
\hline Common & JLA & $24.02 \pm 0.26$ & $2.14 \pm 0.07$ & 0.91 \\
Common & Panstarrs & $23.90 \pm 0.24$ & $2.17 \pm 0.07$ & 0.92 \\
Common & Union2.1 & $23.97 \pm 0.32$ & $2.13 \pm 0.09$ & 0.87 \\
Extra & JLA & $24.33 \pm 0.19$ & $2.20 \pm 0.05$ & 0.96 \\
Extra & Panstarrs & $23.97 \pm 0.25$ & $2.17 \pm 0.07$ & 0.90 \\
Extra & Union2.1 & $23.16 \pm 0.53$ & $1.81 \pm 0.15$ & 0.63 \\
All & JLA & $24.22 \pm 0.15$ & $2.19 \pm 0.04$ & 0.95 \\
All & Panstarrs & $23.89 \pm 0.18$ & $2.15 \pm 0.05$ & 0.91 \\
All & Union2.1 & $23.51 \pm 0.35$ & $1.95 \pm 0.10$ & 0.71 \\
\hline All & Union2.1 without outliers & $23.97 \pm 0.23$ & $2.11 \pm 0.06$ & 0.87 \\
\hline
\end{tabular}

and the peak B-band brightness as

$$
m_{B}=\frac{5}{\ln (10)} \ln (z)+\left(\frac{5}{\ln (10)} \ln \left(\frac{c}{H_{0}}\right)+25-\alpha \cdot X+\beta \cdot C+M_{B}\right) .
$$

\subsubsection{Methodology}

We use $z \leq 0.1$ as our cut-off point. Although this is a slightly arbitrary number, any cut-off up to $z<0.15$ would be valid for the linear relationship to hold. We use $z \leq 0.1$ as a fairly conservative cut-off. This limit is taken to be the one where quasi linear relationship between $\ln (z)$ and $\mu_{B}$ will hold (see eq. 3.3). In order to study the consistencies in the data, we use a simple regression law between the redshift and the supernovae magnitude. We fit the $m_{B} \mathrm{v} / \mathrm{s} \ln (z)$ correlation to a linear model with weights corresponding to $\sigma_{m_{B}}$. As expected from theoretical considerations outlined above, we obtain a relationship following $m_{B}(z)=a+b \times \ln (z)$ and determine the confidence levels on the intercept $a$ and slope $b$ of the regression. Should any statistically significant difference exist from the background cosmology at low redshift, this would be detected in the inconsistencies of the fitted parameters of the linear regression model (especially the slope) between the different datasets and in comparison to theory at a level determined by their respective confidence levels.

\subsubsection{Results}

As described in section 2, we consider three different supernovae datasets that are typically used to constrain the cosmological models. Amongst those datasets some 86 supernovae were found to be common to all datasets at low- $z$, although each has been processed separately between the different groups, and sometimes also observed by different telescopes. To assess whether different processing techniques or observations introduce bias between the datasets, we first look at the properties of the common data points between the different sets.

Figure 3 shows the regression plots of the magnitude $m_{B}$ for the three datasets as a function of $\ln (z)$ for (a) the supernovae common to all datasets, (b) the supernovae that are different between the datasets (called 'Extra') and (c) all the supernovae in each dataset. The regression lines are calculated based on weighted least squares optimization. The values obtained for the linear regression optimization are given in Table 4. The parameter of interest to compare with theory is the slope of the regression line. Therefore we do not highlight the results obtained on the value of the intercept, though it may play a role for higher redshifts.

From fig 3(a), it superficially appears that when considering the 86 data points common to the three datasets, the magnitude values obtained by the different processes do differ significantly. Indeed, the magnitudes determined for the Panstarrs data is consistently lower by 0.19 from the Union2.1 data, and by 0.23 from the JLA data. However, typically simple differences in the intercept can be absorbed by the nuisance parameters $\left(\alpha, \beta, M_{B}\right)$ in cosmological analysis, and do not show 


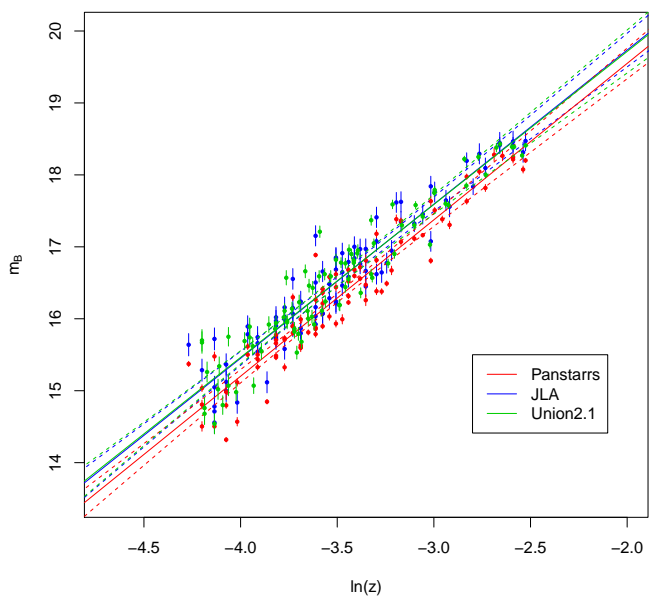

(a)

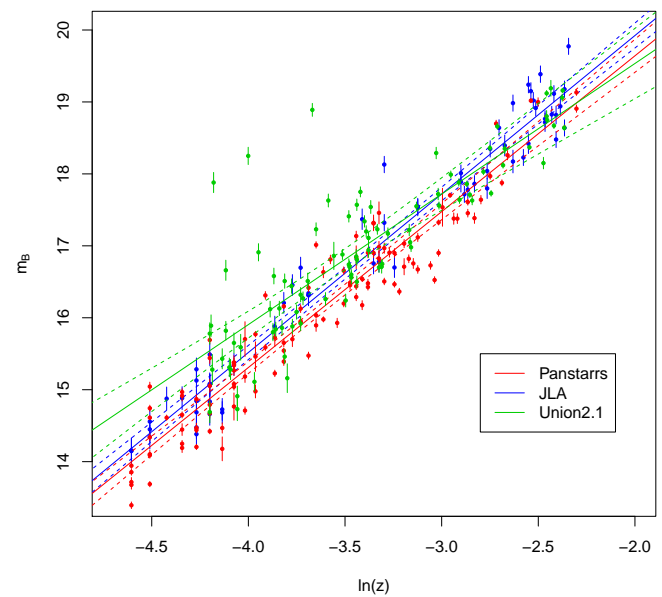

(b)

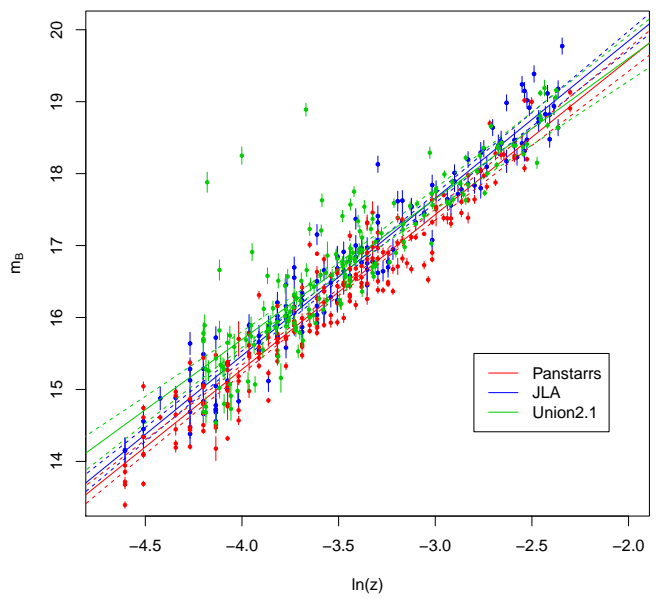

(c)

Figure 3. Linear regression plots of $m_{B}(z)$ as a function of $\ln (z)$ for the three datasets (JLA in blue, Panstarrs in red and Union2.1 in green) for (a) the supernovae common to all datasets at $z \leq 0.1$, (b) the supernovae that are different between the datasets at $z \leq 0.1$, and (c) all the supernovae at $z \leq 0.1$. Regression lines are calculated based on weighted least squares optimization. Continuous lines are the regression line, dashed curves indicate the $2 \sigma$ confidence levels of the regression parameters. A clear difference at the $2 \sigma$ level appears in the regression lines when the extra data points are added to the analysis.

up appreciably in the cosmological parameters. If the slope of the regression line differs considerably between datasets, this would be cause for concern. In this case, as seen in table 4, the analysis shows that the regression slope parameters are very close from one dataset to the next, and within $1 \sigma$ of the calculated regression confidence levels. We also note that the slopes for all three datasets are quite close to the expected theoretical value of $5 / \ln (10)=2.17$. This indicates that the processing followed by the different surveys are consistent and that the cosmological parameters probably would not depend on the processing of the data done by each group or on the observer of the supernova, at least at low redshift.

However, when looking at the data points that have been selected separately in each dataset - 


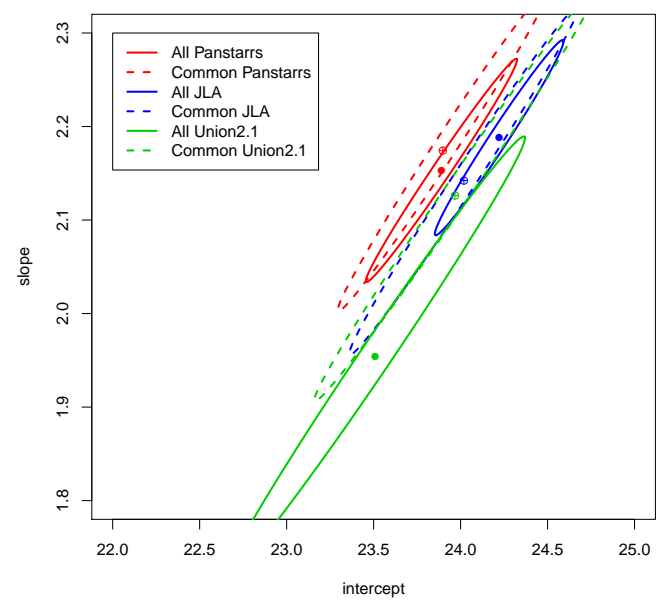

Figure 4. $2 \sigma$ confidence ellipses plotted for the linear regression parameters in the slope v/s intercept space for common data points and all the low- $z$ data $(z \leq 0.1)$ for the three datasets. Best-fit points are marked as crossed circles for the common datasets and filled circles for the full datasets.

those that are not part of the common pool of supernovae - differences in the regression parameters at the $1 \sigma$ level or higher arise, as seen in fig 3(b). These differences are sufficiently large that they translate to a similar level difference in the regression slope between each dataset when all the low redshift $\mathrm{SNe}$ are considered (see fig 3(c)). For example, there is a $1 \sigma$ level difference in the slope obtained for the regression of the data points between JLA and Panstarrs, and a $2 \sigma$ difference between the slopes calculated for Union2.1 and JLA or Panstarrs data. This discrepancy is also seen in Table 4. It appears that more than the choice of the technique used for light curve analysis of supernovae data, the choice of which supernovae to fit is critical in order to get consistent constraints on the cosmological parameters.

The values determined in Table 4 also indicate differences in the quality of the fit between the different datasets. The quantity $R^{2}$ is the coefficient of determination of the regression line, here it represents the square of the correlation between $m_{B}$ and $\ln (z)$. While the common data points for all datasets appear to be equivalently fitted amongst all datasets $\left(R^{2} \approx 0.9\right)$, the extra data points added to the Union2.1 dataset drive the regression parameters away from the ones of JLA and Panstarrs, and lead to a worse regression fit $\left(R^{2} \approx 0.7\right)$. This effect is seen in about ten outlier data points that are clearly located away from the regression line in the upper part of figures $3(\mathrm{~b})$ and (c) between $-4 \lesssim \ln (z) \lesssim-3.5$ or equivalently $0.018 \lesssim z \lesssim 0.03$. The Panstarrs fit for the data, whether using common points or extra appears consistent, with $R^{2}$ values uniformly around 0.9. The difference in slope between Panstarrs and JLA appears driven by the added extra Panstarrs points located at very low redshifts and redshifts close to 0.1. The JLA dataset behaves somewhat better than either Union2.1 and Panstarrs because the fit quality improves as extra data points are added to the common data points, with the $R^{2}$ value increasing to 0.96 and reduced residuals.

Figure 4 summarizes the linear regression results by plotting the $95 \%$ confidence ellipses of the linear regression parameters in the slope $\mathrm{v} / \mathrm{s}$ intercept space for the SNe common to all the datasets and for the entire available data at $z \leq 0.1$. It can be seen from this plot that Panstarrs is $2 \sigma$ away from JLA and Union2.1 whatever the data set considered. However, JLA and Union2.1 are consistent when considering their common data points, but not anymore if all the data points are considered. JLA and Panstarrs are well behaved with respect to the inclusion of additional data, as their $2 \sigma=95 \%$ confidence ellipses are consistent with each other. This is not the case for the Union2.1 dataset, indicating a significant change to the behaviour of the dataset as additional points are included. We also see that the slopes for all common data are consistent with each other and 


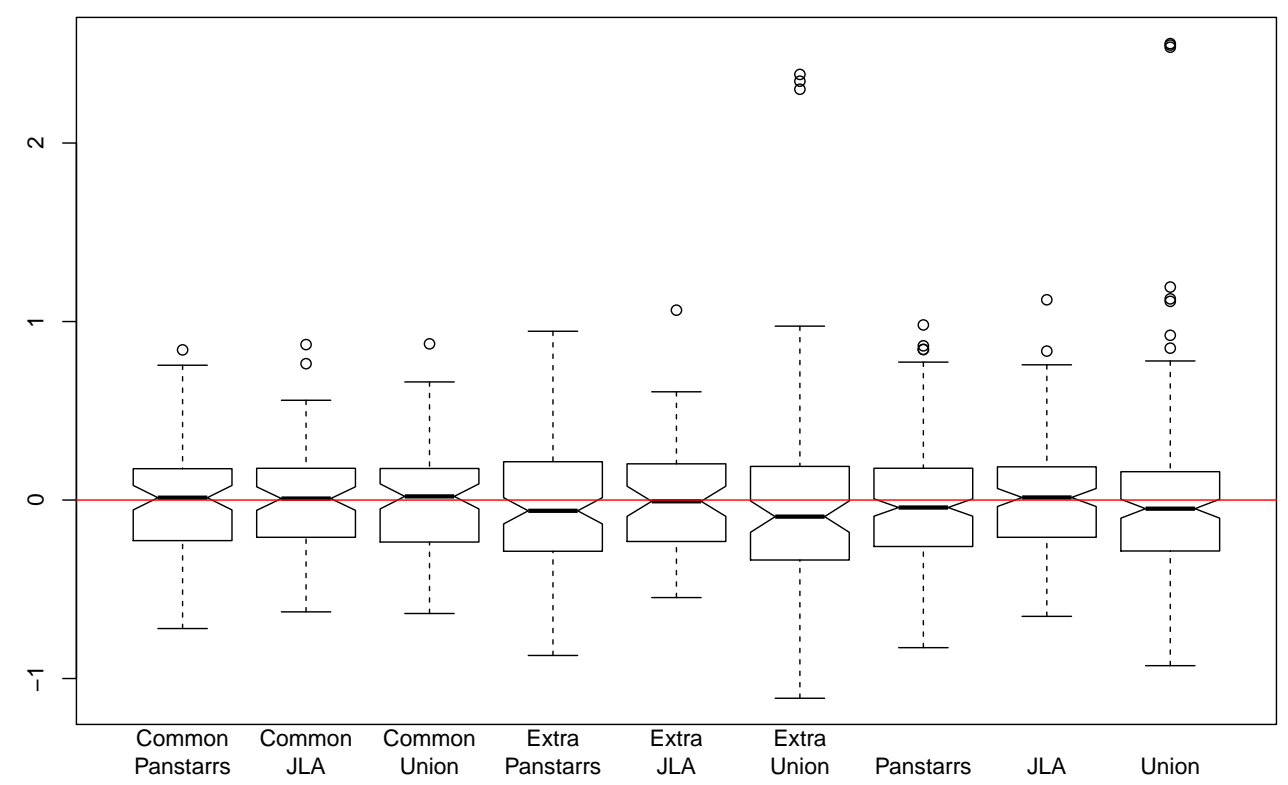

Figure 5. Boxplot of the residual distribution of each fit for the three datasets (Panstarrs, JLA, Union2.1). The residual distribution obtained for the data common to the three sets is consistent and relatively tight. Three strong outlier data points are seen in the Union2.1 Extra subset, while all distributions present a tail of high residual values when considering full datasets.

Table 5. Optimization parameters for the linear regression for the different subsets of Panstarrs.

\begin{tabular}{|l|ccc|}
\hline Subset & Intercept & Slope & $R^{2}$ \\
\hline All data & $23.89 \pm 0.18$ & $2.16 \pm 0.05$ & 0.91 \\
\hline JRK07 (47) & $23.46 \pm 0.37$ & $2.04 \pm 0.10$ & 0.90 \\
CFA3 (84) & $23.87 \pm 0.28$ & $2.15 \pm 0.08$ & 0.91 \\
CFA4 (43) & $23.59 \pm 0.41$ & $2.06 \pm 0.12$ & 0.88 \\
CSP (21) & $24.41 \pm 0.72$ & $2.32 \pm 0.19$ & 0.88 \\
PS1 (7) & $24.36 \pm 0.71$ & $2.24 \pm 0.25$ & 0.93 \\
\hline
\end{tabular}

the theoretical value, while for Union2.1, the full data, due to the effect of the extra data points, is inconsistent with the others and the expected theoretical value.

Boxplots are a way to summarize the distribution of values by drawing the limits of the extreme data and the first and third quartiles surrounding the median value. Figure 5 presents boxplots of the residual distribution obtained for each linear regression model. The residual distributions for the common supernovae of the three sets are consistent and relatively tight. Three strong outlier data points are seen in the Union2.1 subset of extra supernovae data, while all distributions present a tail of high residual values when considering all data points of the sets. This justifies the lower fit quality determined for the Union2.1 data set, and suggests that several outliers are present in this data set. The data points which have residuals above $95 \%$ of the mean of the residual distribution of the fitting model can be considered as potential outliers. Six such points are found (1999gd, 2002hw, $2006 \mathrm{br}, 2006 \mathrm{~cm}, 2006 \mathrm{gj}, 2005 \mathrm{a}$ ) and if they are removed from the analysis, then both the quality of the fit $\left(R^{2}=0.87\right)$ and the values for the intercept and slope of the regression lines are much closer 
to the ones obtained from JLA and Panstarrs analyses as shown in Table 4. However, the slope value still remains more than $1 \sigma$ away from the ones calculated for the JLA dataset, indicating that the differences are not just driven by a few outlier data points, but are present systematically between the sets of supernovae chosen for analysis by the different groups.

As a further check, we obtain the same result by comparing the variance (Fisher-Student test) of the residual populations between the different datasets and for the different linear models. The comparison between the variance of residuals for the linear regressions based on the common data points indicates no statistical difference between the residuals distributions ( $\mathrm{p}$ value $>0.05$ ). This is not the case when comparing the distributions of the residuals for the Union2.1 data set consisting of extra points as well as for the whole data set of low- $z$ values as compared to JLA and Panstarrs. The Fisher-Student test does not show a statistical difference between JLA and Panstarrs but the hypothesis of identical variance between the residuals of Union2.1 and the other datasets should be rejected due to very small p-values (around $10^{-6}$ ). When the 6 outlier data points are removed from the Union2.1 data set, then the Fisher-Student test remains non-significant.

We next separate the datasets into subsets based on the divisions in tables $1,2,3$. We use these subsets to check if these separations could be significantly driving the results with specific biases linked to a single subsample. Each of the JLA, Panstarrs and Union2.1 groups have used their own consistency checks to put together these datasets. Our analyses would serve as a further test of the consistencies within and between these datasets.

For example, the Panstarrs data are separated into 5 subsets (JRK07, CFA3, CFA4, CSP and PS1). Of these, JRK07 is comprised of several datasets put together, as mentioned in section 2. Each subset was used to generate a linear regression which could be compared to the other ones. We note here that the low- $z$ PS1 group has only 7 points (since the PS1 telescope mainly observes higher redshift $\mathrm{SNe}$ ) and should not be considered statistically relevant, although we present results from it for the sake of completeness. The numerical results are shown in Table 5 and the plots presented in Figure 6(a). This panel of the figure compares the regression lines obtained from each subset of the Panstarrs dataset and demonstrates together with Table 5 that no subset is more than $2 \sigma$ away from the other subsets. Figure 6(a) also compares the regressions obtained for each subset with the one obtained for all the data. It can be clearly seen that no subset diverges more than $2 \sigma$ away from the total regression line shown in black and that apart from CSP and PS1 which contain a relatively low number of data points, the errors and confidence levels of the regression are comparable.

The JLA dataset is separated in 7 subsets (SDSS, CT, CfA1, CfA2, O, CfA3, CSP). Similar to the analysis we did for the Panstarrs subsets, we compare the linear regression parameters fitted for each subset. The numerical results are shown in Table 6 and the plots presented in Figure 6(b). Here we compare the regression lines obtained from each subset of the JLA dataset and demonstrate together with Table 6 that no subset is more than $2 \sigma$ away from the other subsets, though the subsets containing few data points appear to have larger confidence levels. Subset SDSS is specific in the fact that it contains a relatively large number of data points (34) but they are all located at comparatively high $z(z>0.04)$, so that their linear regression is not particularly tight, and presents large confidence levels. As in the case of PS1 in Panstarrs, most of the SDSS data is actually for the medium redshift range, so the lower redshift SNe from this set may not be particularly relevant to the low redshift analysis. Again, from the figure 6(b), it can be seen that no subset diverges more than $2 \sigma$ away from the total regression line shown in black.

Finally, 8 subsets are present in the Union2.1 dataset given by the different data releases from various observatories that have performed the supernovae observations over the years. The subsets have a very heterogeneous distribution of data points, one subset containing just 4 data points while the largest subset contains 94 of them. We have assessed whether any of the subsets was biasing the data as a whole. The values obtained for the regression models in this case are shown in Table 7 . The regression slopes are all within $2 \sigma$ of the value for the model that includes all the data points. For the two subsets that contain the least number of data points ( 6 and 4 data points respectively), we note that the best-fits are somewhat different, but as these have large confidence levels, they cannot be expected to drive the trend of the dataset.

Figure 6(c) compares the regression lines obtained from each subset of Union2.1 and shows that 


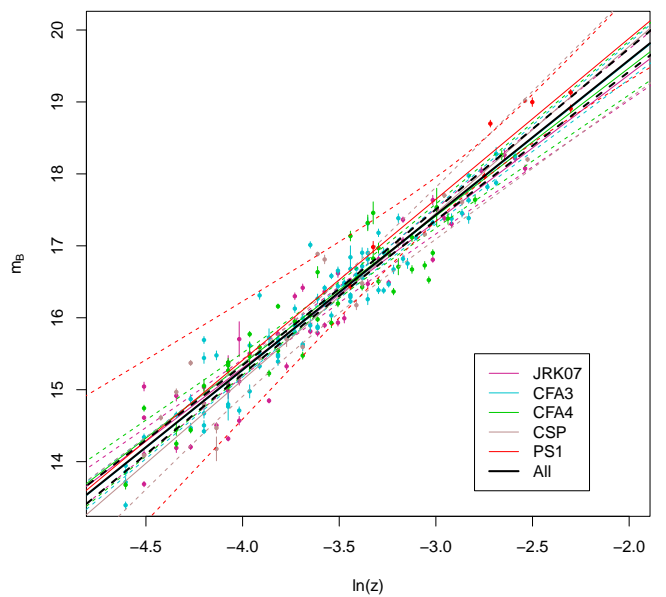

(a)

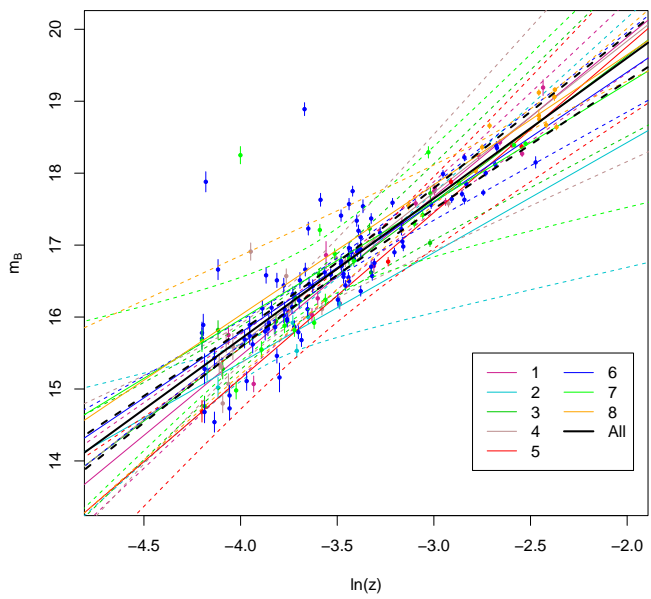

(c)

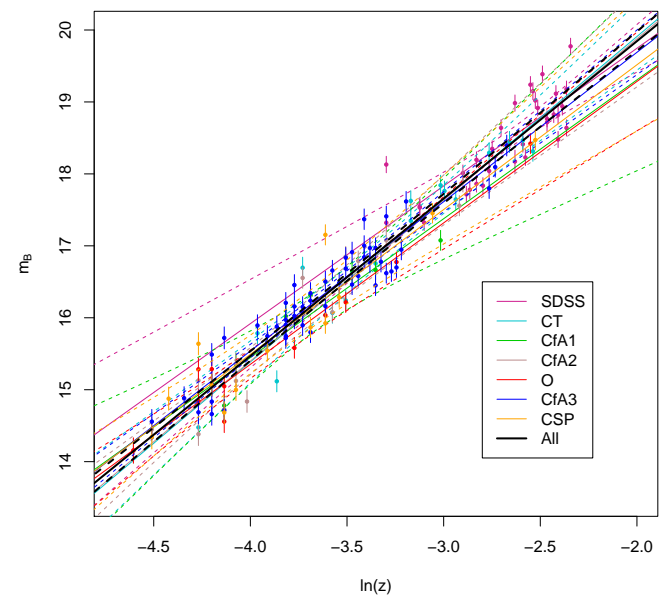

(b)

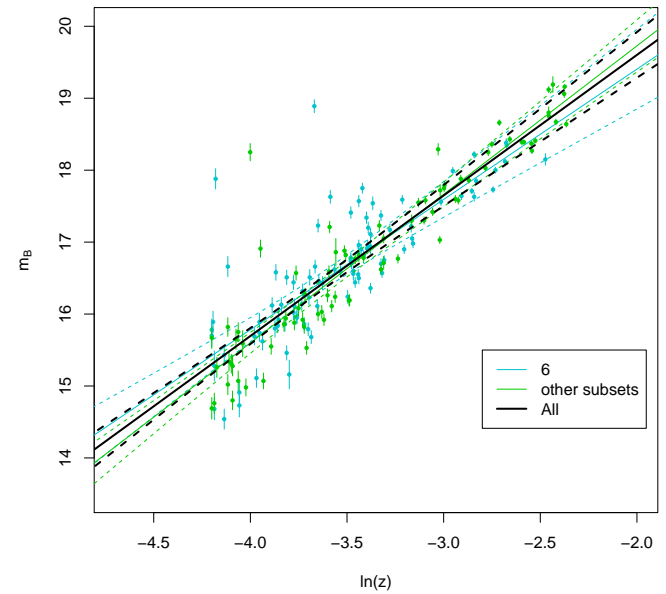

(d)

Figure 6. Regression plots for (a) Panstarrs, (b) JLA ('O' stands for Others), and (c) Union2.1 datasets using separation between the different surveys. Each plot compares the regression lines obtained from each subset of the dataset and shows that no subset is more than $2 \sigma$ away from the others. The black lines represent the regression line and confidence levels for the linear model including all data points. It can be clearly seen that no subset diverges more than $2 \sigma$ away from the total regression line shown in black. Plot (d) compares the regression obtained for subsample 6 with that for the rest of the subsamples of the Union2.1 dataset. While the lines are within $2 \sigma$ of each other, there is a clear trend driven by the subset 6 for a lower slope of the regression line.

no subset is more than $2 \sigma$ away from the other subsets. Though it can be noted immediately that the subsets with the lowest number of data points have very large confidence levels, they are still consistent with the other more constrained regression lines. Again, no subset diverges more than $2 \sigma$ away from the total regression line shown in black. So the behaviour of the data selected within each survey appears consistent.

However, one can see that the subset 6 of Union2.1, which contains the most data points, has a low value for the correlation $\left(R^{2} \approx 0.6\right)$ and the lowest slope for a large subset for any of the 
Table 6. Optimization parameters for the linear regression of the different subsets of JLA.

\begin{tabular}{|l|ccc|}
\hline Subset & Intercept & Slope & $R^{2}$ \\
\hline All data & $24.22 \pm 0.15$ & $2.19 \pm 0.04$ & 0.95 \\
\hline SDSS (34) & $23.57 \pm 0.60$ & $1.91 \pm 0.22$ & 0.67 \\
CT (17) & $24.43 \pm 0.52$ & $2.26 \pm 0.16$ & 0.93 \\
CFA1 (7) & $23.17 \pm 1.06$ & $1.93 \pm 0.29$ & 0.88 \\
CFA2 (15) & $24.36 \pm 0.62$ & $2.24 \pm 0.16$ & 0.93 \\
CFA3 (55) & $23.84 \pm 0.33$ & $2.08 \pm 0.09$ & 0.91 \\
CSP (13) & $23.54 \pm 0.85$ & $2.01 \pm 0.22$ & 0.88 \\
Others (11) & $23.22 \pm 0.61$ & $1.97 \pm 0.16$ & 0.94 \\
\hline
\end{tabular}

Table 7. Optimization parameters for the linear regression of the different subsets of Union2.1. !6 corresponds to the set of all subsamples apart from subsample 6 .

\begin{tabular}{|l|ccc|}
\hline \hline Subset & Intercept & Slope & $R^{2}$ \\
\hline All data & $23.51 \pm 0.35$ & $1.95 \pm 0.10$ & 0.71 \\
\hline $1(17)$ & $24.31 \pm 0.56$ & $2.21 \pm 0.17$ & 0.92 \\
$2(6)$ & $21.49 \pm 1.28$ & $1.53 \pm 0.33$ & 0.80 \\
$3(10)$ & $23.69 \pm 1.02$ & $2.03 \pm 0.27$ & 0.86 \\
$4(15)$ & $24.05 \pm 1.59$ & $2.10 \pm 0.41$ & 0.64 \\
$5(4)$ & $24.38 \pm 0.48$ & $2.31 \pm 0.13$ & 0.99 \\
$6(94)$ & $23.02 \pm 0.60$ & $1.81 \pm 0.16$ & 0.57 \\
$7(18)$ & $22.52 \pm 1.77$ & $1.64 \pm 0.48$ & 0.38 \\
$8(11)$ & $23.27 \pm 0.66$ & $1.81 \pm 0.25$ & 0.83 \\
\hline$! 6(81)$ & $23.86 \pm 0.40$ & $2.06 \pm 0.11$ & 0.82 \\
\hline
\end{tabular}

regression models calculated so far, with a value of 1.81 . If one compares the regression obtained from the subset 6 with that from the rest of the data points (as shown in Table 7), one notices that the regression model obtained for the remaining data points of the Union2.1 dataset is consistent with the model values obtained by JLA and Panstarrs as shown in Table 4. It seems that subset 6 is the most inconsistent set of data with respect to all the other supernovae surveys and is driving the difference between Union, JLA and Panstarrs at low redshift. The respective plots of the models are shown in Figure 6(d) using separation between the survey number 6 and the rest of the surveys. The two plots compare the regression lines obtained from the subset 6 of Union2.1 with the regression line obtained by considering all the other subsets except for 6 . The lines are about $2 \sigma$ away from each other, in addition, there is a clear trend driven by the subset 6 for a lower slope of the regression line, while the rest of the data from the Union2.1 dataset is much more consistent with both JLA and Panstarrs.

Thus we find that, while the subsets in the Panstarrs and JLA datasets are quite consistent with each other and the full datasets, the set 6 of the Union2.1 dataset is almost $2 \sigma$ inconsistent with the other Union2.1 subsets and with the JLA and Panstarrs datasets. It is also inconsistent with the expected theoretical value of 2.17. We note here that subset 6 of Union2.1 is drawn from the same dataset as the CfA3 subset of both JLA and Panstarrs. However, the results from it do not match those from either the JLA subset of CfA3 or the Panstarrs one (as seen from tables 5, 6, 7).

Therefore, from the above analysis we may conclude that the significant differences obtained when comparing the three low- $z$ datasets most likely correspond to a bias introduced by the selection of the supernovae within the different surveys, as it does not appear to stem from the processing techniques. The difference may be driven by the selected data points and could be traced to some extent to the data subset 6 of Union2.1 (drawn from CfA3) which appears to be driving the trends in the regression seen in the Union2.1 dataset. It is not clear by how much, if at all, these differences 


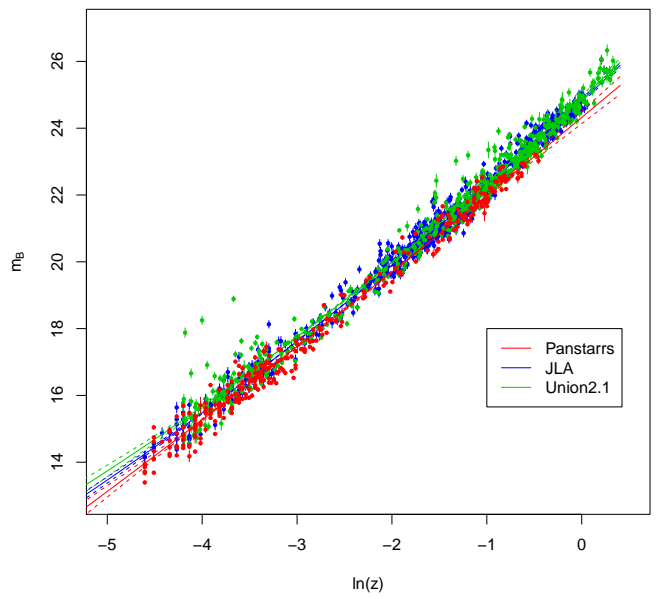

(a)

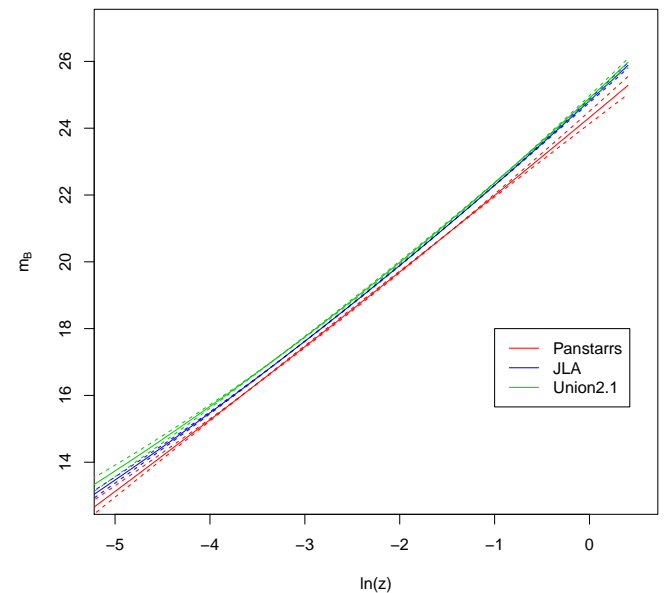

(b)

Figure 7. Nonlinear regression plots of the datasets using all the data from the three data sets. (a) With and (b) without the data points to allow visualization of the regression curves.

in the low redshift datasets translate to cosmological results. This will be studied in section 4 .

\subsection{Nonlinear regression model analysis for all supernovae data}

We now consider the entire datasets for each of Panstarrs, JLA and Union2.1. We note that while low redshift data is essential for calibrating the datasets, the cosmological information in the supernovae Type Ia is really available in the mid to high- $z$ data. The high- $z$ data for Union2.1 is a composite of several different surveys whereas for JLA it is mostly dependent on SNLS and SDSS data, while for Panstarrs it is entirely drawn from the Panstarrs telescope survey.

\subsubsection{Methodology}

When considering redshift values larger than 0.1, the linear relationship of the previous section cannot be used, and needs to be adapted to a non-linear variation of the data. The initial relationship we considered for low- $z$ data was $m_{B}(z)=a+b \times \ln (z)$. To take into account nonlinear regression by adding minimal parameters in a cosmology-blind analysis, we now introduce a third parameter that corresponds to a quadratic term in $\ln (z)$ so that the magnitude to redshift relationship is expressed as $m_{B}(z)=a+b \times \ln (z)+c \times \ln (z)^{2}$. As the initial magnitude to redshift correlation at low- $z$ is almost linear, the parameter $c$ is a minor correction to the relationship and we find that it gives good results for the model fitting in SNe data. We obtain the best fit by using a generalized nonlinear least square fitting algorithm [67, 72]. It is important to remember here that this fit does not take into consideration any cosmological models. It is simply a statistical fit to the data which has no physical or cosmological significance, and thus has no bias towards any specific cosmology.

\subsubsection{Results}

Table 8 presents a summary of all the results for the nonlinear regression fitted parameters. We have separated the data into various subsets to compare models amongst the datasets. The first nonlinear regression analysis was done on the entire data sets for Panstarrs, JLA and Union2.1 without any further processing. From the results of the table, it can be seen that the regression model is relatively good. This can also be seen in Figure 7.

In the table, it can be seen that the parameters calculated for the Panstarrs data are very different from the JLA and Union2.1 data by more than $2 \sigma$. While JLA and Union2.1 parameters are 
Table 8. Optimization parameters for the non-linear regression of the different datasets. Clow refers to the common low redshift data subset, $\mathrm{H}$ to the high redshift dataset.

\begin{tabular}{|c|c|c|c|c|c|}
\hline subset & $\mathrm{N}$ & $a$ & $b$ & $c$ & RSD \\
\hline \multicolumn{6}{|l|}{ All data } \\
\hline JLA & 740 & $24.82 \pm 0.03$ & $2.59 \pm 0.03$ & $0.06 \pm 0.007$ & 0.097 \\
\hline Panstarrs & 310 & $24.32 \pm 0.09$ & $2.36 \pm 0.09$ & $0.02 \pm 0.02$ & 0.082 \\
\hline Union2.1 & 580 & $24.90 \pm 0.04$ & $2.61 \pm 0.05$ & $0.08 \pm 0.01$ & 0.128 \\
\hline \multicolumn{6}{|l|}{ Clow $+\mathrm{H}$} \\
\hline JLA & 674 & $24.83 \pm 0.03$ & $2.61 \pm 0.03$ & $0.06 \pm 0.008$ & 0.094 \\
\hline Panstarrs & 194 & $24.29 \pm 0.10$ & $2.31 \pm 0.11$ & $0.007 \pm 0.02$ & 0.070 \\
\hline Union2.1 & 491 & $24.88 \pm 0.03$ & $2.54 \pm 0.05$ & $0.04 \pm 0.01$ & 0.103 \\
\hline \multicolumn{6}{|c|}{ Clow $+\mathrm{H}(<0.64)$} \\
\hline JLA & 546 & $24.85 \pm 0.06$ & $2.63 \pm 0.06$ & $0.07 \pm 0.01$ & 0.099 \\
\hline Panstarrs & 194 & $24.29 \pm 0.10$ & $2.31 \pm 0.11$ & $0.007 \pm 0.02$ & 0.070 \\
\hline Union2.1 & 383 & $24.67 \pm 0.08$ & $2.30 \pm 0.1$ & $-0.005 \pm 0.02$ & 0.105 \\
\hline \multicolumn{6}{|l|}{$\mathrm{H}$} \\
\hline JLA & 588 & $24.88 \pm 0.03$ & $2.78 \pm 0.06$ & $0.15 \pm 0.03$ & 0.090 \\
\hline Panstarrs & 108 & $24.28 \pm 0.24$ & $2.34 \pm 0.37$ & $0.04 \pm 0.13$ & 0.076 \\
\hline Union2.1 & 405 & $24.90 \pm 0.03$ & $2.67 \pm 0.08$ & $0.15 \pm 0.05$ & 0.104 \\
\hline \multicolumn{6}{|c|}{$\mathrm{H}(<0.64)$} \\
\hline JLA & 460 & $25.18 \pm 0.1$ & $3.22 \pm 0.15$ & $0.3 \pm 0.06$ & 0.095 \\
\hline Panstarrs & 108 & $24.28 \pm 0.24$ & $2.34 \pm 0.37$ & $0.04 \pm 0.13$ & 0.076 \\
\hline Union2.1 & 297 & $24.4 \pm 0.16$ & $1.75 \pm 0.28$ & $-0.21 \pm 0.11$ & 0.106 \\
\hline \multicolumn{6}{|c|}{ Large subsets } \\
\hline JLA & 668 & $24.81 \pm 0.03$ & $2.60 \pm 0.03$ & $0.07 \pm 0.008$ & 0.093 \\
\hline Panstarrs & 197 & $24.24 \pm 0.1$ & $2.25 \pm 0.11$ & $0.001 \pm 0.02$ & 0.076 \\
\hline Union2.1 & 369 & $24.8 \pm 0.07$ & $2.52 \pm 0.09$ & $0.06 \pm 0.02$ & 0.124 \\
\hline
\end{tabular}

closer, they still are around $1 \sigma$ different and thus not as consistent as they could be. This difference could be driven by the low- $z$ data difference that was discussed in the previous section as it is clearly noticed that for high- $z$ data, the curves appear to be superposed for the three datasets, e.g., in the Figure 7(b).

It is to be noted that on all the fits calculated for the Panstarrs data, the parameter $c$ added for the nonlinear regression is never contributing significantly to the fit (as can be seen from its estimated value which is typically much smaller than the other two parameters), and therefore although it is reported here for consistency, we do not attach much meaning to it. This shows that the behaviour of Panstarrs data is different from JLA and Union2.1 as it is not different from a linear regression even including the high- $z$ data points, whereas a better fit is obtained for JLA and Union2.1 when including the non linear term. This difference could be due to the lower number of data points at high- $z$ for Panstarrs, since the current dataset presents a cut-off around $z=0.64$. Thus, as the Panstarrs data does not go to very high redshifts, the quadratic term containing $c$ may not be very strongly required for fitting this dataset. However, one can see from Table 8 that $c$ remains a significant parameter in the non-linear fits of especially the JLA dataset even when the high- $z(>0.64)$ data points are removed. If this tendency to linearity is confirmed by future, higher redshift Panstarrs measurements, it would have strong implications on the cosmology constraints. At the moment we are not well-placed to present any clear judgement on this.

Interestingly, among the three datasets, the one which presents the least weighted residual standard deviation, RSD, is Panstarrs. For a regression fit, the RSD is the root mean square weighted 


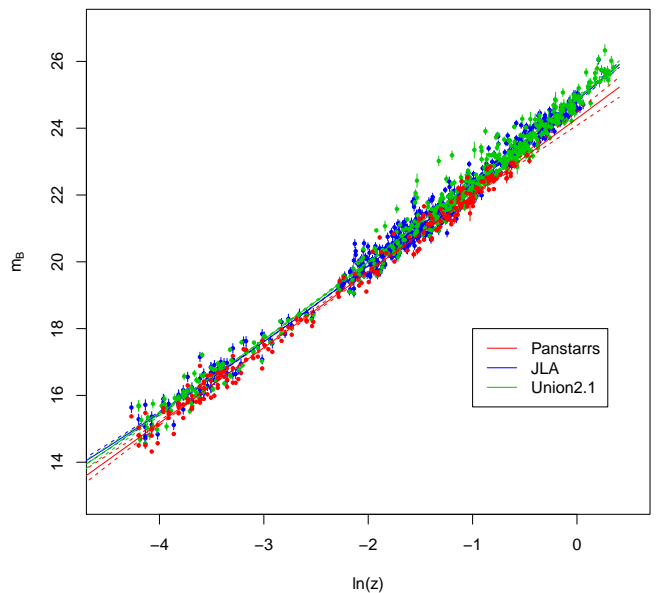

(a)

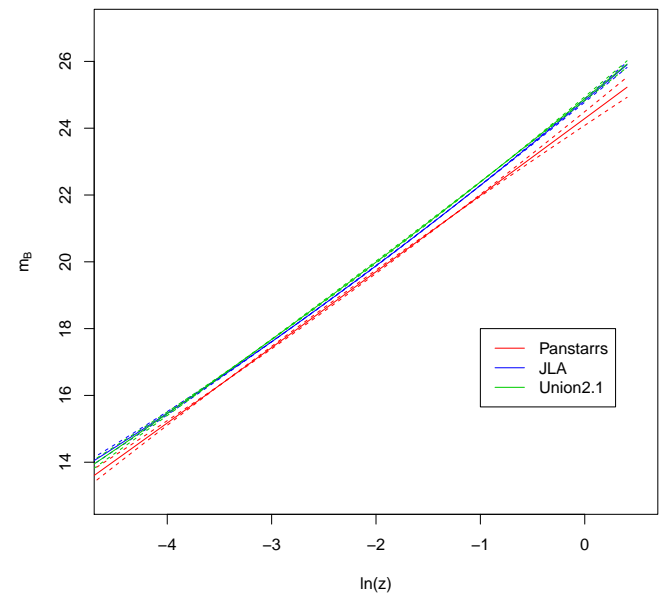

(b)

Figure 8. Nonlinear regression plots of the datasets using common low- $z$ and all high- $z$ data from the three data sets. (a) With and (b) without the data points to allow visualization of the regression curves.

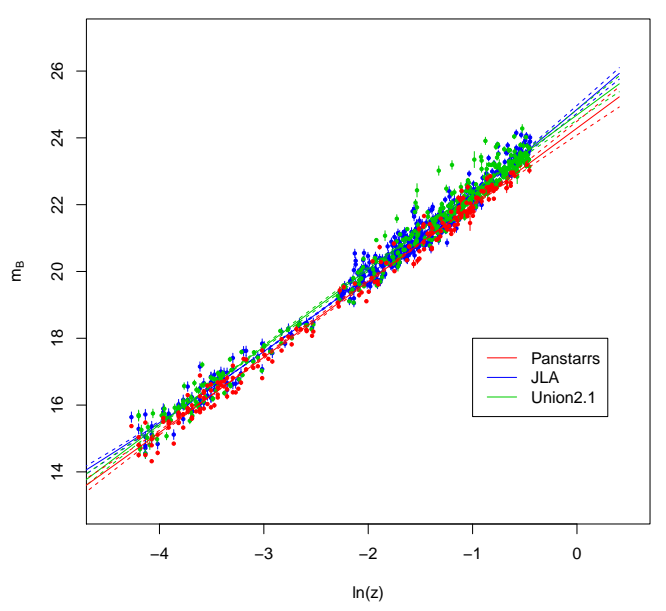

(a)

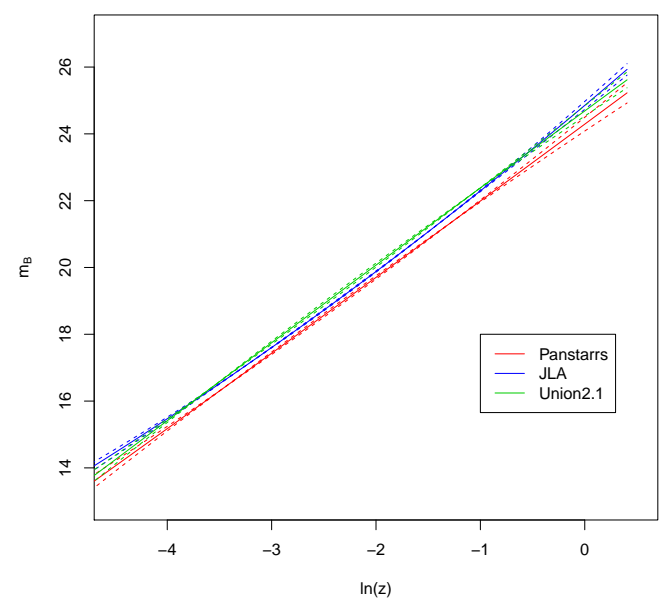

(b)

Figure 9. Nonlinear regression plots of the datasets using common low- $z$ and high- $z$ data $(<0.64)$ from the three data sets. (a) With and (b) without the data points to allow visualization of the regression curves.

residual sum of squares for the model, $\sqrt{\sum \frac{1}{n-p}\left(m_{B}-m_{B_{f i t}}\right)^{2}}$, where $n$ is the number of data points and $p$ the number of free parameters. Thus, small RSD indicates a good fit to the data and tighter residual distribution. The Panstarrs data, even though it is the smallest dataset, or perhaps because of it, appears to be the most consistent within itself.

We can now assess the influence of the high- $z$ data by considering the common low- $z$ data points for every data set, since we demonstrated in Section 3.2 that the fitted models are consistent for the common low- $z$ data points. In this case, as we see in fig 8 , the values for the parameters do not change much, but the errors are tightened as this common subset of data is more consistent amongst all data sets. However, the slopes for JLA and Union2.1 are now a bit further away from each other, showing 


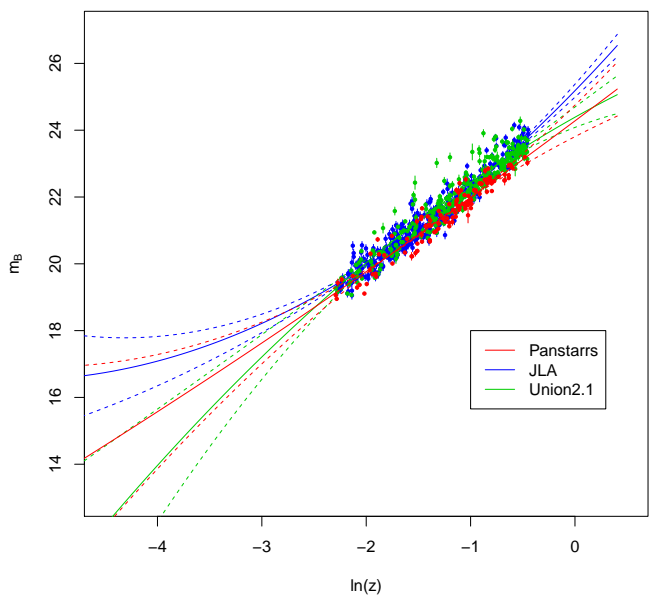

(a)

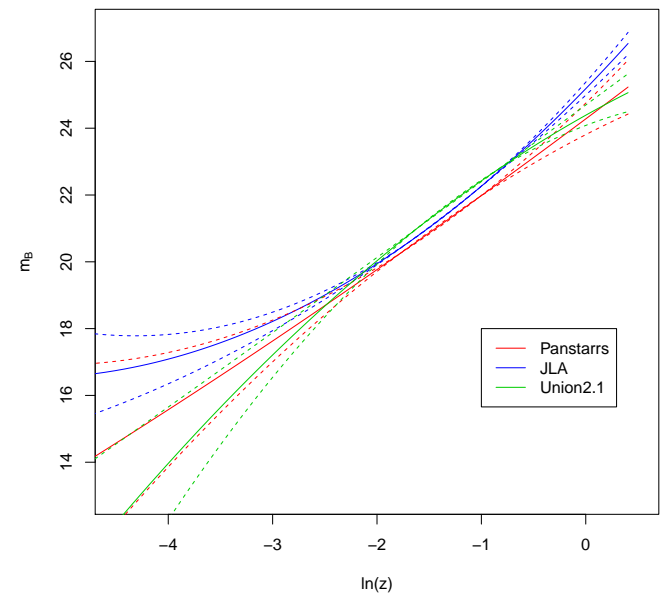

(b)

Figure 10. Nonlinear regression plots of the datasets using only high- $z$ data $(<0.64)$ from the three data sets. (a) With and (b) without the data points.

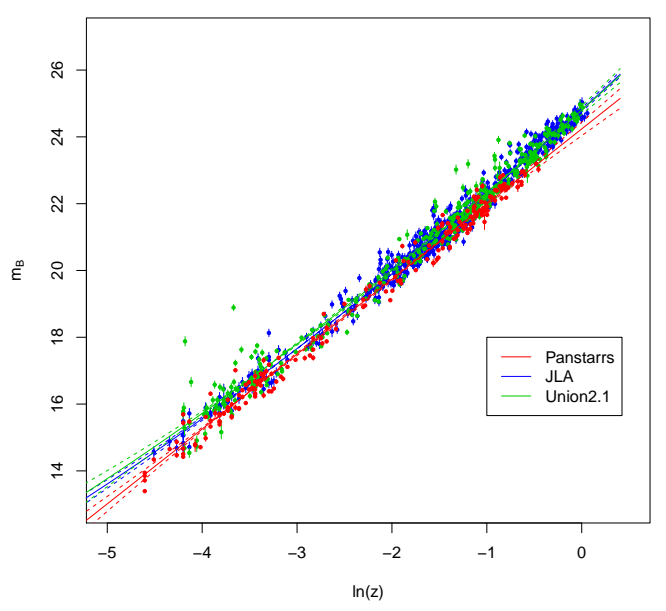

(a)

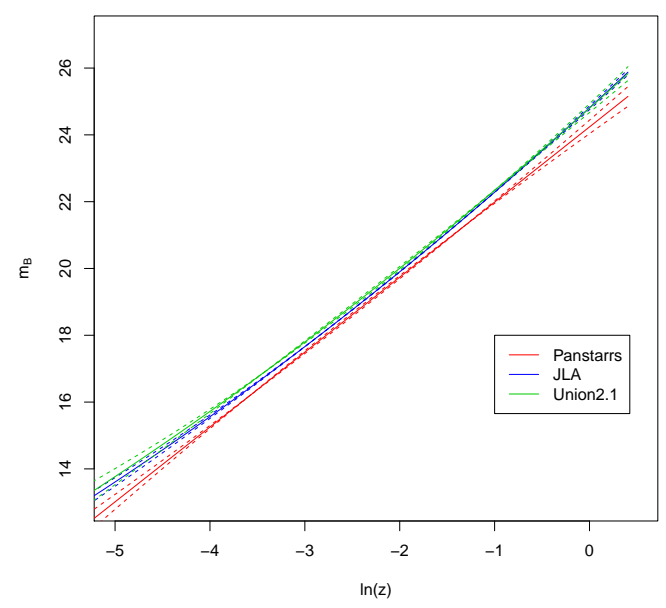

(b)

Figure 11. Nonlinear regression plots of the datasets using the largest data sets within Panstarrs, JLA and Union2.1. (a) With and (b) without the data points.

that consistency in low- $z$ data does not enhance the consistency of the global nonlinear regression, probably due to a smaller number of supernovae data points below $z=0.1$ as compared to higher redshift data.

The Panstarrs data only goes up to a redshift of $z<0.634$. If we now remove the high- $z$ data above the Panstarrs upper limit by introducing a cut-off at $z=0.64$ for all datasets, this will give us an idea if the results from Panstarrs are different from those of JLA and Union2.1 mainly due to it having a smaller redshift range or not. From figure 9, we see that apparently the difference between Panstarrs and the other two remains. However, from table 8, we see that the linear parameter $b$ is now very consistent between Panstarrs and Union2.1, and the quadratic term $c$ also is closer, while JLA 
remains different from the other two datasets. It is tempting to assume that, since it is mainly the gradient $b$ and possibly the quadratic term $c$ which represent the changes in cosmology (rather than the intercept $a$ ), the Union2.1 and Panstarrs data, when cut-off at $z<0.64$, might give cosmological results consistent with each other. However, we should remember that in a quadratic expression, the first and second terms do not simply represent the intercept and gradient of the equivalent linear regression, and the three parameters $a, b, c$ are degenerate with each other. So two datasets can be said to behave equivalently only when all three parameters $a, b, c$ have similar values.

It is singular to note from Table 8 that when considering only the high- $z$ datasets, JLA and Union2.1 appear to be more consistent, while strong differences arise between them when introducing the cutoff at $z<0.64$. This further supports the conclusion that the choice of data points, especially within the Union2.1 dataset, could be the main driver for determining the constraints for cosmological parameter fitting. We also note that the Panstarrs dataset probably remains to this date too small to draw strong conclusions from its cosmological constraints.

We also look at just the high redshift data at $0.1<z<0.64$ for three datasets in fig 10. These regressions are extremely different from each other, and without the low redshift data acting as an anchor, the JLA data in particular shows a highly non-linear behaviour. The Panstarrs data remains the one with the lowest deviance, but the values of the parameters for all three cases are very different, and the interesting consistency between Union2.1 and Panstarrs when using common low- $z$ and high$z(<0.64)$ data is now gone. This illustrates the importance of the low redshift data in normalizing the entire dataset.

Finally, the regression based on the largest subsets of each dataset is also considered. This allows us to see if the largest subsets are consistent or not with the general behaviour of each dataset. For Panstarrs, we select CfA3 and Panstarrs-1 samples for a total of 197 data points; for JLA, we select CfA3, SDSS and SNLS for a total of 668 data points, and for Union2.1, we use subsets number 6, 8 , 14 , and 15 for a total of 369 data points. Using these subsets that correspond to the majority of the data points in each dataset, we see in figure 11 that the fit parameters are practically identical to the ones that were obtained using the whole dataset for JLA, and reasonably close to the whole dataset results for Panstarrs and Union2.1.

Figure 12 summarizes the regression results by plotting the $2 \sigma$ confidence ellipses of the quadratic regression parameters in the $b \mathrm{v} / \mathrm{s} a$ space, and in the $b \mathrm{v} / \mathrm{s} c$ space for the entire datasets, for the sets with common low- $z$ data, for the sets with common low- $z$ and high- $z$ cut-off, and for the large subsets. It can be seen from this plot that Panstarrs is typically more than $2 \sigma$ away from JLA and Union2.1 whatever the data subset considered. However, JLA and Union2.1 are consistent at $2 \sigma$ when considering all the data. One may note that when considering the entire redshift range, the influence of the common and extraneous data points at low- $z$ plays a minimal role. However, choosing an upper limit cut-off of $z<0.64$ (the highest redshift for Panstarrs) changes completely the confidence levels for Union2.1. Although the intercept remains different from Panstarrs, both the slope and the quadratic term come very close to the Panstarrs value. This points out once more the strong sensitivity of this dataset to the choice of the data points included. This also indicates the possibility that the cosmological constraints from Union2.1 and Panstarrs may be slightly more consistent in the limit $z<0.64$, while the JLA should retain its discrepancy with Panstarrs irrespective of the subsets considered.

Figure 13 represents the boxplot distribution of the standardized residuals for each subset. One can see that there is a systematic presence of potential outliers on the upper part of the supernovae curves. This corresponds to supernovae for which the magnitude is overestimated with respect to their redshift, indicating a population of supernovae that appear to have a lower emission flux than expected. This probably does not reflect an observational bias since brighter supernovae should be detected at the same rate. It could be due to a difference in the absorbing medium between the source and the observer or other effects changing the brightness of the supernovae considered, like different properties of their host galaxies (see e.g., [73], [74]).

Similar to the low redshift data, we may now attempt to check the effect of the different subsets in the data. In the case of JLA and Panstarrs, the high redshift data is typically taken from a few telescopes (only one in the case of Panstarrs), but Union2.1 has several subsets at high redshift, 


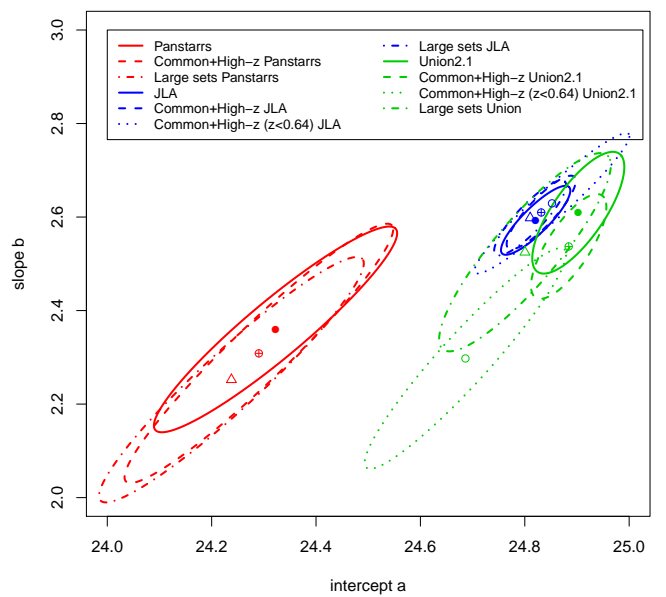

(a)

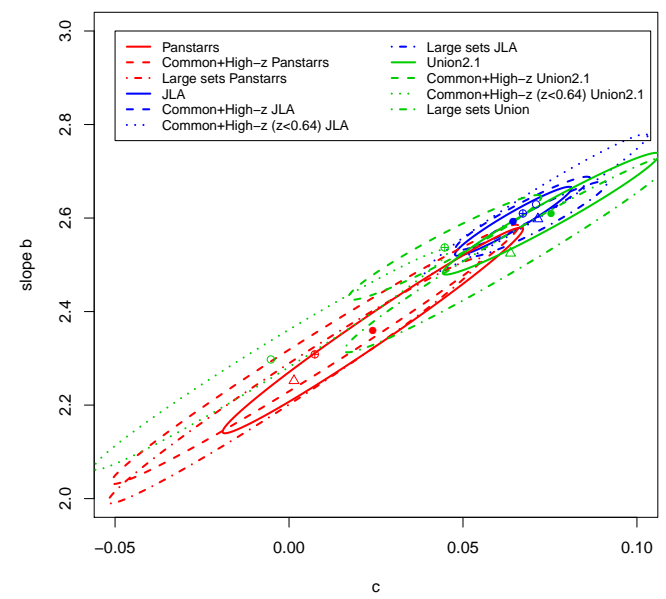

(b)

Figure 12. $2 \sigma$ confidence ellipses plotted for the non-linear regression parameters in (a) $b \mathrm{v} / \mathrm{s} a$ space and (b) $b \mathrm{v} / \mathrm{s} c$ space, for the entire dataset (filled circle for best-fit), common low- $z+$ high- $z$ data (crossed circle), common low- $z+$ high- $z(<0.64)$ (empty circle), and for the largest subsets (triangle) for the three datasets.

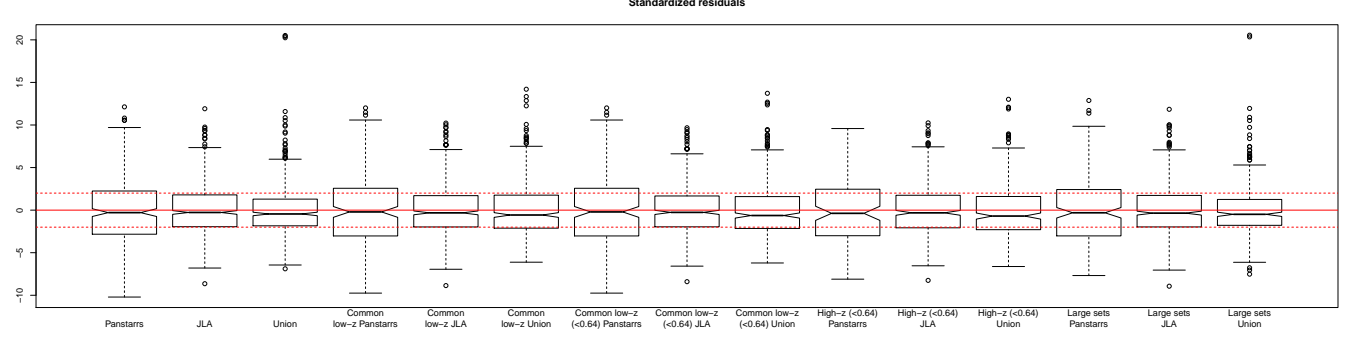

Figure 13. Boxplots of the standardized residual distributions for each nonlinear model of the supernovae data. Horizontal lines indicate the $2 \sigma$ levels.

with various quantities of $\mathrm{SNe}$ in them. In the figure 14, we show the residuals for the different subsets in Union2.1. We note here that the largest high redshift datasets $(14,15)$ are actually quite well-behaved being centred around the mean with few outliers. The effect of the largest low redshift dataset (i.e., subset 6) is somewhat mitigated by better behaviour from the high redshift data. So we do not expect the results to be drastically different between the data subsets. The figure 15 shows the standardized residuals for each subset of Union2.1. Except for a few outliers the different subsets appear to be reasonably consistent with each other. Thus, although this dataset is quite diverse, the different subsets are fairly well-chosen. The analysis thus does not show a specific behaviour by subset for the Union2.1 data. In the figure 15(b), one can appreciate the variations in residual that are visible for low and high redshifts, presenting a visible non-normal distribution and heteroscedasticity as noted earlier in section 3.1. This is further illustrated by the quantile-quantile comparison between the residuals and the normal distribution shown in 15(c), which indicates the over-representation of large positive residuals. These variations may play a role in the cosmological parameter fitting. A similar analysis was done using the Panstarrs and JLA data subsets, for which just a couple of different observatories have measured the high- $z$ supernovae. None of these analyses show an indication that a specific set of data points particularly drives the resulting models. This demonstrates that the multiple data subsets appear broadly consistent with one another, even though over smaller redshift 


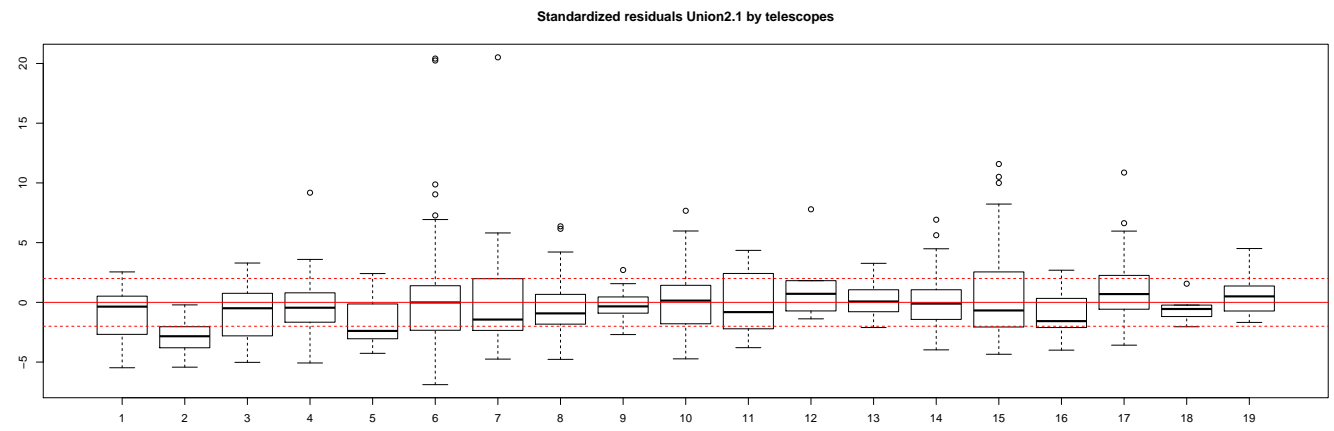

Figure 14. Boxplots of the residual distributions for each subset of Union2.1 data. Horizontal lines indicate the $2 \sigma$ levels.

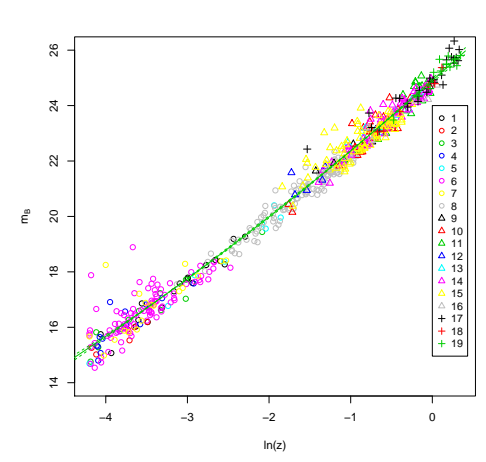

(a)

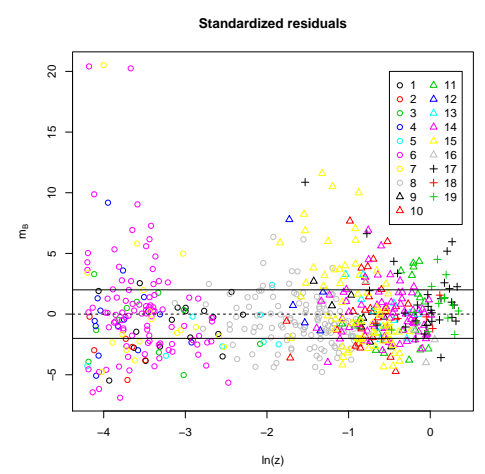

(b)

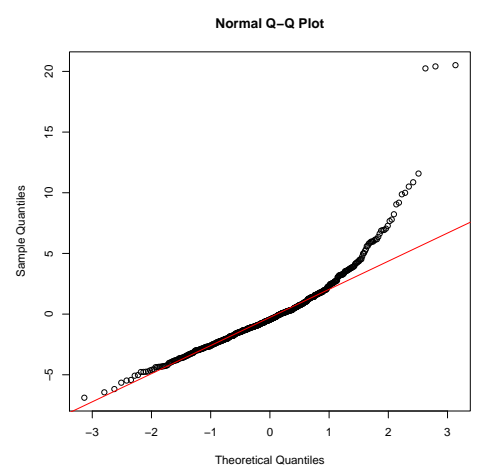

(c)

Figure 15. Study of subset behaviour of Union2.1. (a) Non-linear fit, (b) standardized residuals, and (c) associated quantile-quantile plot comparing the residuals and normal distributions. The curves and lines indicate the $2 \sigma$ levels.

ranges, the Union2.1 sample in particular shows some subset-dependence (e.g., subset 6 drives the data at low- $z$ ).

From the above analyses in linear and non-linear regimes, several points of interest should be noted.

- Firstly, as has been noted elsewhere from cosmological reconstruction, the Union2.1 and JLA data appear statistically consistent with each other, while the Panstarrs is $2 \sigma$ away from either. However, if the data is cut-off at $z<0.64$ which is the upper limit of Panstarrs data, Union2.1 and Panstarrs now appear to be possibly more consistent with each other, while JLA retains its difference. It is not clear if this discrepancy is simply due to the fact that Panstarrs has less data, and is at lower redshifts than the others.

- In the low redshift data, the data subset 6 of Union2.1 appears to be rather discrepant from the other subsets of Union2.1 and with JLA and Panstarrs data, as well as with the expected theoretical behaviour. This behaviour may or may not result in drastic differences in cosmology since low redshift data acts mainly as an anchor, but it is worth noting especially since the other data subsets appear fairly consistent with each other.

- The Union2.1 dataset appears to be especially sensitive to which data subsets are chosen. We find the most variance within this dataset and the statistical results appear to change significantly depending on the subset of data used. The other two datasets do not show such 
strong proclivity for change. This may be due to the fact that the Union2.1 dataset is created as a mixture of several different datasets observed by various different telescopes at different times, thus it is the most heterogeneous of the three datasets. This may or may not have an effect on the cosmological results.

- We also note, that, although the Panstarrs dataset has the least number of data points and goes up to only moderate redshifts, it is the most consistent of the three datasets and least prone to change upon subset choice. It also shows the least heteroscedasticity, and has the least RSD of the three. In the future, a more complete Panstarrs dataset, with more data points at high redshift, may actually end up being the most stable.

- Finally, we note that, barring Panstarrs, the other two datasets show some strong heteroscedasticity. This variation in residual variance as a function of redshift may lead to a larger influence of the lower error points over the best fitting parameters and their confidence levels, and may result in a bias in the cosmological results. Of course, the analysis will depend both on the errors and the distance from the fits, and typically, given that the spread in errors are fairly small, the distance from the fit would drive the results. The errors may still be capable of changing results, especially when the degrees of freedom of the system increase, making the fit tighter.

\section{Cosmology}

We are now in a position to verify our findings from the statistical blind analysis by reconstructing cosmological models from these datasets.

\subsection{Methodology}

The data is in the form:

$$
\mu(z)=5 \log _{10}\left(\frac{c(1+z)}{H_{0}} \int_{0}^{z} \frac{d z_{1}}{h\left(z_{1}\right)}\right)
$$

with $h(z)$ given by

$$
h^{2}(z)=\Omega_{0 r}(1+z)^{4}+\Omega_{0 m}(1+z)^{3}+\left(1-\Omega_{0 r}-\Omega_{0 m}\right)(1+z)^{3(1+w)},
$$

for a flat universe with a constant equation of state dark energy $w$ and luminosity distance measured in $10 \mathrm{pc}$. The matter and radiation densities at present are denoted by $\Omega_{0 m}$ and $\Omega_{0 r}$ respectively, and $H_{0}$ is the Hubble constant. It should be noted that at the redshifts considered, the radiation density $\Omega_{0 r}$ is negligible, and also that the only effect of the parameter $H_{0}$ is as an additive constant. Thus marginalizing over $H_{0}$ does not affect the SNe results and indeed $H_{0}$ can be absorbed into the additive nuisance parameter $M_{B}$. The flatness of the Universe can be assumed from CMB, and is a required assumption to reduce the degeneracies between the different densities. The radiation density is also calculated from CMB, e.g., from [20], even though it has little effect. As mentioned earlier in sec 2, the constant equation of state is not a very realistic model for dark energy, but the supernova data alone does not have sufficient accuracy to constrain the rate of change of equation of state of dark energy, and adding other datasets to the analysis could potentially add the biases of those datasets to the reconstruction. As the main objective of this paper is not to constrain cosmology, but rather to examine the consistencies and inconsistencies of the SNe data, we therefore use the constant equation of state model and assume a flat universe for all our cosmological reconstruction.

We use a standard $\chi^{2}$ minimization analysis, and a Markov Chain Monte Carlo analysis for the confidence levels, with $\chi^{2}$ defined as

$$
\chi^{2}=\sum_{i=1}^{N_{\text {data }}}\left(\frac{\mu_{i}\left(\Omega_{0 m}, w, \alpha, \beta, M_{B}\right)-\mu_{f i t}\left(z_{i}\right)}{\sigma_{i}\left(\alpha, \beta, \sigma_{\text {int }}\right)}\right)^{2},
$$

where the parameters of interest are the matter density $\Omega_{0 m}$ and the equation of state of dark energy $w$. The nuisance parameters $\alpha, \beta, M_{B}$ (which includes $H_{0}$ ) are marginalized over and we present our 
Table 9. Best-fit and $1 \sigma$ confidence levels on the cosmological parameter $w$ (with $\Omega_{0 m}=0.3$ ) for various subsets of data. Clow refers to the common low redshift data subset, $\mathrm{H}$ to the high redshift dataset.

\begin{tabular}{|l|rcc|}
\hline & \multicolumn{3}{|c|}{$w$} \\
\hline Subset & Panstarrs & JLA & Union2.1 \\
All data & & & \\
Clow $+\mathrm{H}$ & $-1.334_{-0.117}^{+0.076}$ & $-1.090_{-0.024}^{+0.056}$ & $-1.147_{-0.045}^{+0.045}$ \\
& $-1.306_{-0.116}^{+0.095}$ & $-1.078_{-0.059}^{+0.060}$ & $-1.118_{-0.047}^{+0.051}$ \\
Clow $+\mathrm{H}(<0.64)$ & $-1.306_{-0.116}^{+0.095}$ & $-1.044_{-0.071}^{+0.070}$ & $-1.099_{-0.055}^{+0.055}$ \\
& & & \\
W/O outliers & $-1.303_{-0.113}^{+0.096}$ & $-1.032_{-0.050}^{+0.058}$ & $-1.128_{-0.044}^{+0.043}$ \\
Large subsets & $-1.259_{-0.113}^{+0.110}$ & $-1.026_{-0.059}^{+0.059}$ & $-1.027_{-0.053}^{+0.054}$ \\
W/O subset 6 & - & & $-1.225_{-0.053}^{+0.053}$ \\
\hline
\end{tabular}

results in the $\Omega_{0 m}-w$ parameter space. To simplify matters further, we at first utilize a reduced number of parameters for this exercise where we fix $\Omega_{0 m}=0.3$ (commensurate with the Planck values [20]) and only constrain $w$. This gets rid of the potential degeneracy between $\Omega_{0 m}$ and $w$. Later, we also obtain the results in the $\Omega_{0 m} \mathrm{v} / \mathrm{s} w$ parameter space.

\subsection{Results}

Several subsets of the data may be considered, as evinced by the analysis of the previous section. After considering the full dataset, one may consider only the common dataset for low redshift in conjunction with all the high redshift data. One may also truncate the high redshift data at $z<0.64$, which is the highest redshift for the Panstarrs sample. For the JLA sample, considering only the common low- $z$ $\mathrm{SNe}$, this cut-off results in a sample of $546 \mathrm{SNe}$, while for Union2.1 it is a sample of 386 SNe. The logic behind this step is to assess whether the sizeable difference between Panstarrs and the other two datasets is simply due to the former reaching only medium redshift ranges. We may form a tighter data sample by throwing out all SNe that are outliers in the residual distributions for the SNe, as seen in fig 13. We select these potential outliers by determining which residuals are $2 \sigma$ away from the average residual, using the standard deviation of the residuals distribution as described in Section 3.2. For the Panstarrs, JLA and Union2.1 data, this means removing 16, 36 and 22 SNe respectively from the full datasets. We may also, rather arbitrarily, look at how the cosmological results change if only the largest low and high redshift subsamples are used. For Panstarrs we use the low redshift CfA3 data subset and the Panstarrs-1 sample at higher redshifts, giving a sample of 197 SNe. For JLA this would involve the SDSS at low to medium, and SNLS at medium to high redshifts, with the CfA3 sample at low redshifts for anchoring. This results in a dataset with $668 \mathrm{SNe}$. In the case of Union2.1, this subset contains the sample 6 at low redshift, sample 8 at low to medium redshift, and samples 14,15 at medium to high redshifts, resulting in a total of 369 SNe. This step would let us know how important the different subsamples are, if the results are being driven only by the largest subsets, or if the smaller subsets with a few $\mathrm{SNe}$ each also have a role to play in the reconstruction. Also, in the case of the Union2.1 data, particularly, one may redo the cosmological analysis without the subset 6 (from the CfA Year 3 sample), which, though the largest low redshift dataset, also shows the most discrepant behaviour in comparison to both other Union2.1 subsets, and the JLA and Panstarrs samples. We attempt to study all these various possibilities and compare the cosmological constraints obtained in each case.

Table 9 shows the best-fit and $1 \sigma$ values of $w$ for the full dataset and all the subsamples outlined above. The figure 16 shows the likelihood in $w$ for the three datasets in the different scenarios. 


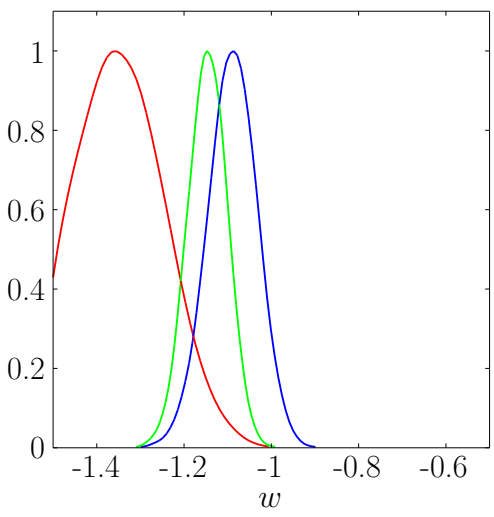

(a)

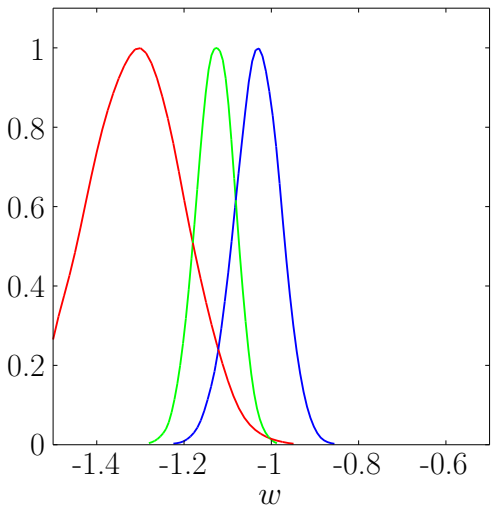

(d)

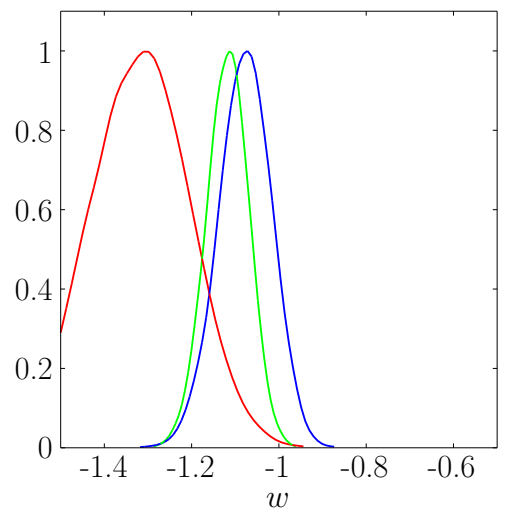

(b)

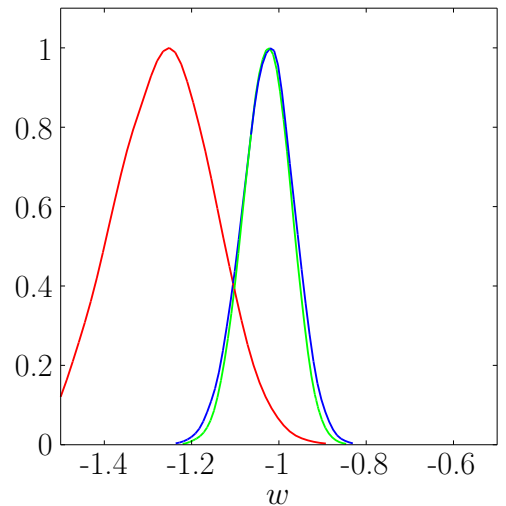

(e)

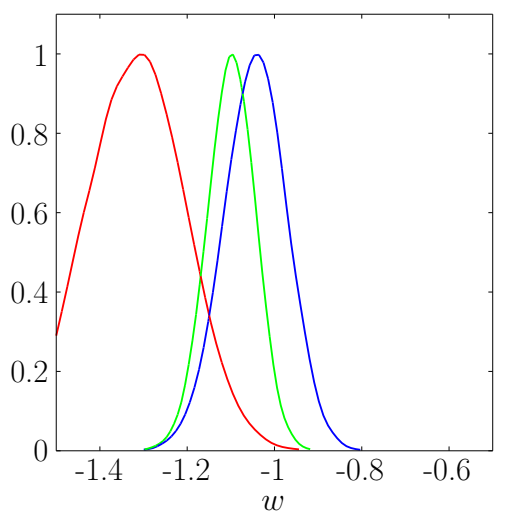

(c)

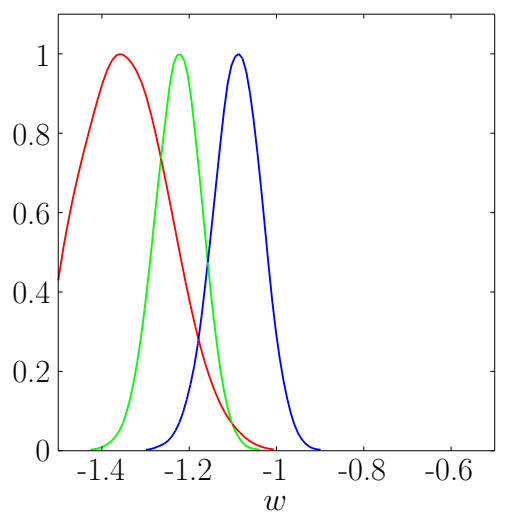

(f)

Figure 16. Probability distribution of the parameter $w$ (with $\Omega_{0 m}=0.3$ fixed) for Panstarrs (red), JLA (blue) and Union2.1 (green) supernova Type Ia data. Panel (a) is for the entire datasets, panel (b) is for common low- $z$ data + high- $z$ data, panel $(\mathrm{c})$ is for common low- $z$ data + high- $z(<0.64)$ data (with Panstarrs remaining the same as in (b)), panel (d) shows results for data without the outliers of regression, panel (e) considers the largest low and high- $z$ subsets for each sample, panel (f) shows full datasets for JLA and Panstarrs, and Union2.1 without the data subset 6 which showed anomalous behaviour.

We find that, the Panstarrs and JLA samples are quite insensitive to changes in the dataset. For various subsamples, the best-fit remains very similar for Panstarrs, with a slightly larger change for the largest subsets case, which may well be the effect of too few (197) data points, while the errors remain nearly the same. This shows that the Panstarrs data, as expected from the statistical analysis, is very consistent within itself, even though the results from it are about $2 \sigma$ different from the other two datasets. The JLA data is also very consistent, with the best-fit values varying only slightly, though the errors show a slightly bigger variation. Again this result is commensurate with what we found in the statistical analysis. As expected from the previous section, the Union2.1 data shows the maximum variation in the best-fit between the data subsets, about twice as much as the others. This may be due to the fact that the Union2.1 dataset is comprised of many different datasets, and therefore it is not as homogeneous as the other two, in which efforts have been made to keep at least the high redshift data more homogeneous. This also points to the fact that if we had a single, high quality source for low redshift data, this could lead to more stable cosmological constraints, which would be even less dependent on data selection. We also note that all three datasets show similar trends for the different subsets. For the full dataset, Panstarrs has the lowest $w$, and JLA the highest 
Table 10. Best-fit and $1 \sigma$ confidence levels on the cosmological parameters $\Omega_{0 m}, w$ for various subsets of data. Clow refers to the common low redshift data subset, $\mathrm{H}$ to the high redshift dataset.

\begin{tabular}{|l|ccc|ccc|}
\hline & \multicolumn{3}{|c|}{$\Omega_{0 m}$} & \multicolumn{3}{c|}{$w$} \\
\hline Subset & Panstarrs & JLA & Union2.1 & Panstarrs & JLA & Union2.1 \\
\hline \multirow{2}{*}{ All data } & $0.164_{-0.164}^{+0.055}$ & $0.250_{-0.056}^{+0.104}$ & $0.291_{-0.042}^{+0.063}$ & $-1.064_{-0.134}^{+0.263}$ & $-0.990_{-0.182}^{+0.228}$ & $-1.097_{-0.147}^{+0.166}$ \\
Clow + H & $0.169_{-0.169}^{+0.057}$ & $0.216_{-0.070}^{+0.116}$ & $0.280_{-0.043}^{+0.068}$ & $-1.047_{-0.138}^{+0.271}$ & $-0.908_{-0.148}^{+0.246}$ & $-1.080_{-0.155}^{+0.178}$ \\
& & & & & \\
Clow H $(<0.64)$ & $0.169_{-0.169}^{+0.057}$ & $0.302_{-0.052}^{+0.132}$ & $0.331_{-0.030}^{+0.088}$ & $-1.047_{-0.138}^{+0.271}$ & $-1.096_{-0.355}^{+0.177}$ & $-1.211_{-0.289}^{+0.098}$ \\
& & & & & & \\
W/O outliers & $0.170_{-0.159}^{+0.067}$ & $0.235_{-0.066}^{+0.113}$ & $0.305_{-0.035}^{+0.057}$ & $-1.044_{-0.133}^{+0.267}$ & $-0.914_{-0.160}^{+0.232}$ & $-1.159_{-0.159}^{+0.154}$ \\
Large subsets & $0.197_{-0.115}^{+0.132}$ & $0.202_{-0.090}^{+0.124}$ & $0.259_{-0.071}^{+0.133}$ & $-1.055_{-0.163}^{+0.294}$ & $-0.849_{-0.111}^{+0.256}$ & $-0.977_{-0.186}^{+0.270}$ \\
W/O subset 6 & - & - & $0.332_{-0.018}^{+0.033}$ & - & $-1.347_{-0.153}^{+0.040}$ \\
\hline
\end{tabular}

(and closest to $\Lambda \mathrm{CDM}$ ) while Union2.1 has an intermediate value. For the common low- $z$ subsample, all three best-fit values for $w$ increase. For the case with the cut-off at $z<0.64$, both the Union2.1 and JLA best-fit increases, thus this cut-off does not make the three datasets any more consistent. For the case without outliers, the $w$-values increase as well, but by a lesser amount than in the common case for Union2.1, and by a greater amount (though by very little) for JLA and Panstarrs. For the case with the large subsets, the $w$ values increase further, and the Union2.1 result especially increases by a larger amount to bring it very close to the JLA result. The Union2.1 dataset gives a lower $w$ when the uncharacteristic subset 6 is taken out, which makes it slightly more consistent with the Panstarrs dataset, but still noticeably separated from it in $w$-space. Thus, while the three datasets are inconsistent with each other, they are quite consistent in their inconsistency, and changing the selection of SNe using various criterion does not make them match each other.

We now remove the constraint on $\Omega_{0 m}$ and let it be a free parameter. The resultant analysis will naturally be affected by the degeneracy between the parameters $\Omega_{0 m}$ and $w$ and the constraints obtained may consequently be poorer. In table 10, we show the best-fit and $1 \sigma$ values in the different scenarios for $\Omega_{0 m}$ and $w$. Fig 17 shows the 1 and $2 \sigma$ confidence levels in the $\Omega_{0 m}-w$ parameter space for (a) the entire Panstarrs, JLA and Union2.1 datasets, (b) the common low- $z$ data + high- $z$ data, (c) the common low- $z$ data + high- $z$ data cut-off at $z=0.64$, (d) the data without the $2 \sigma$ outliers in the regression analysis, (e) the case where only the largest datasets in the low and high redshift ranges are considered for each sample, and (f) the specific case where the anomalous subset 6 is subtracted from Union2.1. We have seen in the statistical analysis and also the cosmological single parameter analysis that the Panstarrs entire dataset looks quite different than the JLA and Union2.1 data, being $2 \sigma$ away from both, while JLA and Union2.1 also appear to be about $1 \sigma$ away from each other. The same trend is seen in the two-parameter cosmological results as well, in figure 17(a), with the Panstarrs confidence levels $2 \sigma$ away, and JLA and Union2.1 about $1 \sigma$ away from each other. We also see that the Union2.1 data appears to give a somewhat higher $\Omega_{0 m}$ and is furthest from the cosmological constant value of $w=-1$ for the equation of state. The Panstarrs data gives much lower $\Omega_{0 m}$ than is usually expected from other observations such as large scale structure, and JLA appears to be closest to the standard cosmological constant model with an amount of matter density that matches the large scale structure constraints. Trends similar to this result have been seen in previous works, albeit with additional information from $\mathrm{CMB}$ and $\mathrm{BAO}$ data.

We now see the effect of using only the common data subset in the low- $z$ data, in fig 17(b). Though the results change in each dataset, the amount by which they change is rather different. The change to common low- $z$ data appears to affect Panstarrs least of all and the results are very similar 


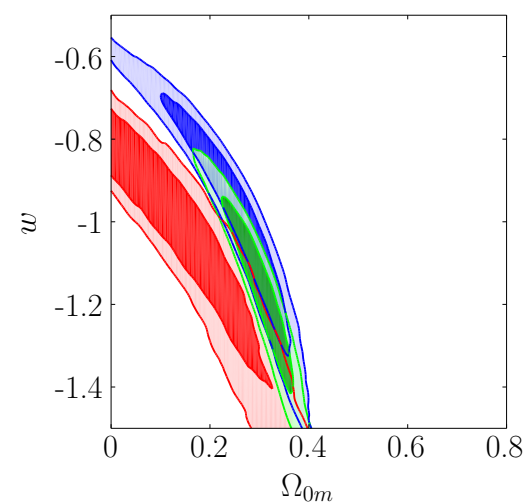

(a)

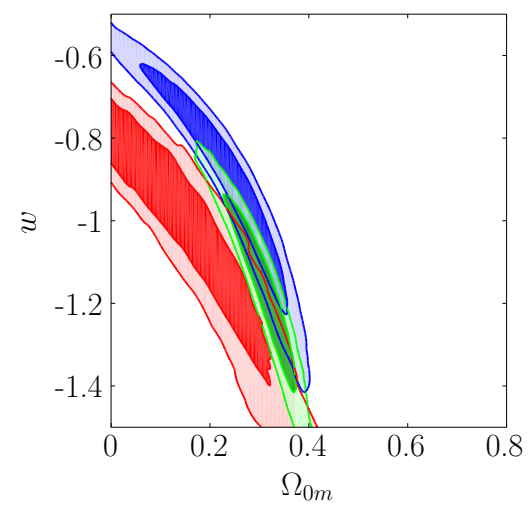

d

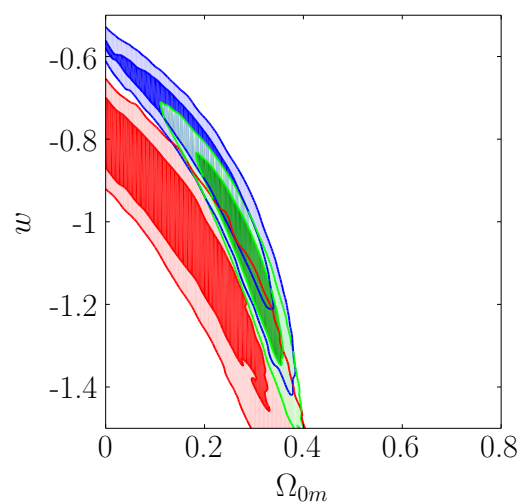

(b)

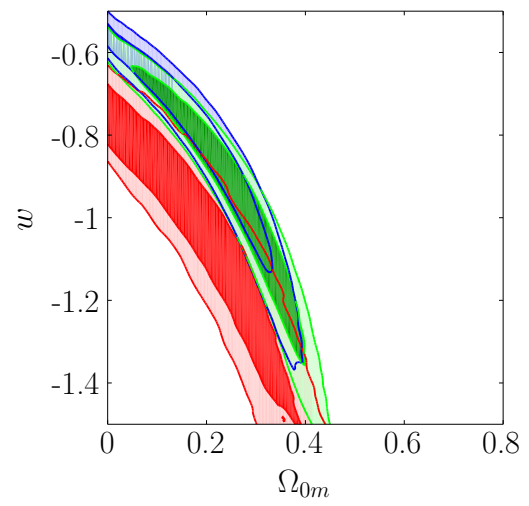

(e)

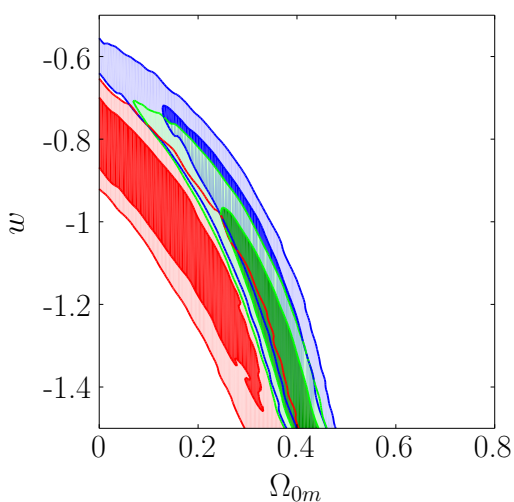

(c)

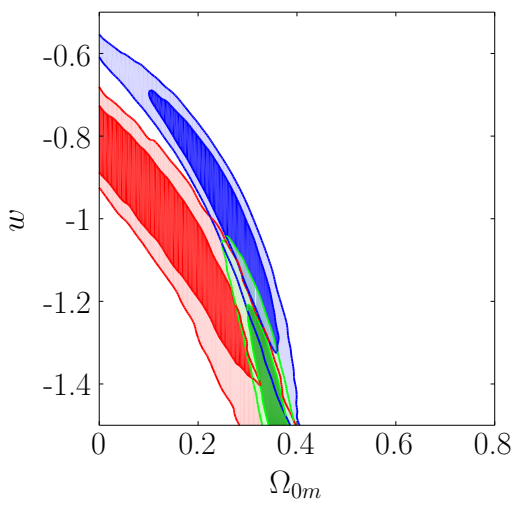

(f)

Figure 17. $1-2 \sigma$ confidence levels in $\Omega_{0 m}-w$ parameter space for Panstarrs (red), JLA (blue) and Union2.1 (green) supernova Type Ia data. Panel (a) is for the entire datasets, panel (b) is for common low- $z$ data + high- $z$ data, panel $(\mathrm{c})$ is for common low- $z$ data + high $-z(<0.64)$ data, panel $(\mathrm{d})$ is for data without the outliers of regression, panel (e) is for the largest low and high- $z$ subsets for each sample, and panel (f) shows full datasets for JLA and Panstarrs, and Union2.1 without the data subset 6 which showed anomalous behaviour.

to that for the full dataset. JLA appears to have a rather large change with $\Omega_{0 m}$ decreasing and $w$ increasing, moving it away from both the cosmological constant and the other datasets. This is unlike the results of both the statistical analysis and the single parameter cosmological analysis. Union2.1 sees a moderate decrease in $\Omega_{0 m}$ and similar increase in $w$. Thus the JLA appears to be most affected by changing low redshift data, but this does not make it more consistent with the other datasets.

Next, we cut off the JLA and Union2.1 data at redshift $z=0.64$, based on the highest redshift SNe available for Panstarrs. We find in fig 17(c) that the confidence levels for both JLA and Union2.1 increase, as expected, since the sample size has shrunk. For JLA, $\Omega_{0 m}$ increases while $w$ decreases, thus keeping it still away from Panstarrs. In the case of Union2.1 as well, a similar trend is noted. Although Panstarrs and Union2.1 now are slightly more consistent at $2 \sigma$, due to the larger confidence levels for Union2.1, substantive inconsistency remains.

In the panel (d) of the figure, where we get rid of the outliers of the regression analysis, we see that once again, Panstarrs results are quite stable while JLA results change quite majorly and Union2.1 rather less. In any case, the various data selections made on the datasets do not resolve the inconsistencies of the fitted cosmological parameters. 
From fig 17(e), we see that the Panstarrs data does show some change in the case where the largest subsets are considered, possibly because the number of data points has reduced drastically, by about a third of the total number of SNe. JLA and Union2.1 datasets also change rather majorly, but the discrepancy between the datasets remains. We note here, that, unlike in the single parameter case, JLA and Union2.1 do not become noticeably more consistent with each other.

When the subset 6 is removed from the Union2.1 dataset in the final panel, this results in somewhat smaller errors, but not more consistency between datasets, again unlike the single parameter case where the Union2.1 result had moved towards the Panstarrs values. In fact, here the cosmological parameters retrieved from Union2.1 become strongly inconsistent with the other datasets, with a much lower value of $w$ and much higher value of $\Omega_{0 m}$. This may be due to the fact that subset 6 is the largest low redshift sample, and without it, the low redshift data may be inadequate in anchoring the full dataset, the effect of which is felt more strongly for a larger number of parameters. The smaller errors in this case as opposed to the full dataset reflects the fact that the subset 6 is rather inconsistent with the rest of the data, and subtracting it from the dataset reduces the intra-sample tension. Of course, smaller errors do not necessarily reflect the accuracy of the result, the result may well become biased due to lack of anchoring.

We note here that the consistencies in the results should also reflect on the standardization parameters, $\alpha, \beta, M_{B}$. Among these, $M_{B}$ being a simple additive, has least effect on the cosmological results, and is the most stable to subset changes. We study the parameters $\alpha, \beta$ for the case where we vary both $\Omega_{0 m}, w$, the results are very similar for the other case, since these parameters are practically independent of cosmology. For all three datasets, we find that $\alpha$ is quite stable to changes within the dataset. For JLA, the best-fit varies within $\alpha=0.137-0.141$, for Union2.1, $\alpha=0.121-0.128$, and for Panstarrs, $\alpha=0.146-0.153$, all with $1 \sigma$ errors of around $\sim 0.01$. The variation in $\alpha$ within the subsets thus falls within the error bars. For $\beta$, the JLA variation is $\beta=3.10-3.31$, for Union2.1 it is $\beta=2.44-2.58$, and for Panstarrs, $\beta=3.76-4.00$, with errors of the order of $\sim 0.15$. For this parameter, we note that the variation is of the same order as the $1 \sigma$ errors for JLA and Union2.1, and somewhat larger for Panstarrs. These results are commensurate with the results obtained in [41, 43, 44], with the Panstarrs result rather closer to that obtained for the case where $\sigma_{\text {int }}$ is dominated by color dispersion. Once again, the values obtained for each dataset are somewhat different to those obtained for the other datasets. The somewhat large variation in $\beta$ may be an effect of host galaxy properties, or of intrinsic scatter, and this difference needs to be studied in more detail, as well as its potential effects on cosmological results.

The basic finding in this analysis is the same as that of the statistical analysis and the single parameter cosmological analysis- we find that using various subsets of the SNe samples does not necessarily lead to more consistency between the three data samples, and the inconsistency between them remains. Worryingly, using different subsets does change the results noticeably for both JLA and Union2.1 samples, while the Panstarrs dataset, despite being smaller and at lower redshifts, is extremely stable to such changes. The Union2.1 data shows this variability in the previous analyses as well, and its behaviour may be attributed at least partly to the heterogeneity in this dataset. However, the JLA data had appeared to be quite well-behaved and stable to such changes in previous analyses, whereas here large differences are noted. We note here that our results for the single parameter case is quite similar to those obtained in [32], and also that our results for the consistency in the JLA data between the different telescope subsamples is equivalent with this paper. However, it appears that for the two-parameter case, the JLA data is sensitive to the degeneracies in the $\Omega_{0 m}-w$ parameter space. Since the SNe data measures the total energy density, increasing $\Omega_{0 m}$ and decreasing $w$ has the same effect for it as decreasing $\Omega_{0 m}$ and increasing $w$, thus data that look very similar can give rather different results based on this non-unique behaviour of the parameters. The JLA data appears to be particularly susceptible to this degeneracy. The reason for this variability in JLA data in the two-parameter analysis may stem from the differences explored in sec 3.1. We saw there that the Panstarrs data has the most homogeneous error distribution, and that JLA shows strong trends of heteroscedasticity. The cosmological analysis depends on a product term, containing the inverse of the errors of the individual SNe, and the spread in the distribution around the magnitude fit. The dispersion in magnitude being rather larger than the spread in errors, it typically drives the results. 
However, the spread in errors should also affect the results to some extent. As we increase the number of parameters, and therefore the degrees of freedom, the dispersion in magnitude becomes smaller if the fit becomes tighter, and the spread in the errors now may become relatively more significant.

To study this effect further, we look at the residuals of the fit for JLA in the two cases where $\Omega_{0 m}$ is fixed and when it is free. We find that the residuals at best-fit appear to be slightly tighter in the case with two free parameters. If we now look at the different subsets of the JLA sample, for the single parameter fit, the variation of the residuals between datasets is low because of reduced degrees of freedom. The two-parameter fit has more flexibility and therefore smaller median residuals for each subset, but larger changes in residual variance between subsets. This divergence is further enhanced by the non-linear $z$-dependence of the magnitude errors resulting in a noticeable difference in the results for different data subsets. For Panstarrs, a similar variation in the residuals can be seen, but as it has more homogeneous errors, this behaviour is not enhanced by the errors, and the fits remain relatively stable. Therefore, the heterogeneous errors in the JLA data result in a somewhat more unstable fit when the number of parameters is increased. Adding other types of data that complement SNe data may be one way to limit this behaviour, as seen in fig 16 and table 9, JLA results are very stable if the value of matter density is known, i.e., the degrees of freedom are reduced. Another potential method would be to model the non-linearity of the magnitude errors and include this information in the parameter fitting [71]. Thus the heteroscedasticity of the data may have a distinct effect on the cosmological results, and we should be careful when interpreting such results.

\section{Conclusions}

In this work, we have studied three SNe type Ia datasets available at present, the Panstarrs, JLA and Union2.1 samples. We have first looked at the data from a purely statistical point of view, without considering any cosmological information. We then do a cosmological analysis, constraining first the single parameter $w$, and then the two parameters $\Omega_{0 m}, w$. We present our findings below.

- Simple linear or quadratic regression fits to the data show that the three SNe samples have divergent behaviours and this can be traced to the use of different SNe in each analysis. Thus the choice of SNe appears to make a real difference in the statistical results. These differences are not easily reconciled, and the Panstarrs, JLA and Union2.1 datasets remain inconsistent with each other no matter which data subset is considered. In the cosmological section, these statistical results are corroborated.

- We demonstrate that the Panstarrs data is most homogeneous in its residuals and has the fewest outliers in the regression analysis. It also is very stable when considering different subsets. The Union2.1 data is most heterogeneous, it is strongly affected by choosing different data subsets in the statistical analysis, as well as having a large anomalous subset (sample 6) at low redshift. The JLA data is apparently not affected by subset selection in the simple statistical analysis, but the errors $\sigma_{m_{B}}$ of JLA do not follow a normal distribution and show strong clustering.

- We find that Panstarrs, the dataset drawn from the most homogeneous sample, with a single data subsample at high redshift, performs best in the cosmological fits. The Union2.1 data, drawn from a total of 19 different sources, appears to be less stable than the others. Although enough surveys now exist at high redshift with sufficiently high quality data that it is not necessary to use many surveys in conjunction, the same is not true at low redshift. There we still do not have a single survey with high quality data that could replace the various data sources of varying quality currently used. Since homogeneity of data appears to be important for reliable analysis, we suggest that the presence of such a homogeneous low redshift sample would improve the overall quality of cosmological reconstruction.

- Significant heteroscedasticity in the magnitude errors, as in the case of JLA, appears to lead to a less stable cosmological fit which is dependent on the subset used for analysis, especially for a higher number of parameters. The Panstarrs dataset, on the other hand, with its more homogeneous errors, is comparatively impervious to the data subset selection. If we are to use data 
with strongly heterogeneous errors, we should either model the errors along with the cosmological parameters, or add other datasets to reduce the number of degrees of freedom. A dataset with homogeneous error residuals, such as Panstarrs, would be more stable in constraining a

larger number of parameters.

In conclusion, we find that the currently available SNe datasets are somewhat inconsistent with each other (at up to $2 \sigma$ confidence level). Further data in the future will be able to shed light on this inconsistency. We note that a single, high quality dataset at low redshift may go some way towards increasing the robustness of cosmological reconstruction. Data with homogeneous error residuals tends to be more immune to changes in the data subsets, thus leading to more conclusive parameter estimation. Although the Panstarrs dataset is too small now to draw cosmological conclusions from, it is stable to small changes and consistent within itself. Therefore future data releases from Panstarrs may lead to stronger cosmological constraints. The ideal supernova dataset would be one which is drawn from the least number of samples, potentially one at high redshift, and one at low redshift, with a strongly homogeneous distribution of errors. Cosmological reconstruction from such a supernova sample would be robust and serve as a good complement to other available data. Combining disparate supernova samples requires careful consideration of the systematics involved in the reduction and fitting techniques for SNe data. We note that certain systematics, such as the intrinsic dispersion and the effect of host galaxy properties, could bear closer scrutiny, and we expect to return to the systematics of supernovae in more detail in future work.

\section{Acknowledgements}

UA was supported in this project by the DST Young Scientist Program of SERB, India. The authors would also like to thank the ChemCam team at IRAP, Toulouse for the use of the hyperion2 cluster for some of the calculations of this paper.

\section{References}

[1] A. G., Riess et al., , "Observational Evidence from Supernovae for an Accelerating Universe and a Cosmological Constant", Astron. J. 116 (1998) 1009.

[2] S. Perlmutter et al., , "Measurements of Omega and Lambda from 42 High-Redshift Supernovae", Astroph. J. 517 (1999) 565.

[3] V. Sahni \& A. A. Starobinsky, "The Case for a positive cosmological Lambda term", Int. J. Mod. Phys. D 9 (2000) 373.

[4] P. J E. Peebles \& B. Ratra, "The Cosmological constant and dark energy", Rev. Mod. Phys. 75 (2003) 559.

[5] T. Padmanabhan, "Cosmological constant: The Weight of the vacuum ", Phys. Rep. 380 (2003) 235.

[6] V. Sahni, "Dark matter and dark energy", Lect. Notes Phys. 653, (2004) 141.

[7] V. Sahni, "Cosmological Surprises from Braneworld models of Dark Energy", astro-ph/0502032.

[8] E. J. Copeland, M. Sami \& S. Tsujikawa, "Dynamics of dark energy", Int. J. Mod. Phys. D 15 (2006) 1753

[9] J. A. Frieman, M. S. Turner \& D. Huterer, "Dark Energy and the Accelerating Universe", Ann. Rev. Astron. Astroph. 46 (2008) 385.

[10] R. Durrer and R. Maartens, "Dark Energy: Observational and Theoretical Approaches", ed. P Ruiz-Lapuente, Cambridge UP, (2010) pp. 48 - 91; arXiv:0811.4132.

[11] S. Tsujikawa, "Dark Matter and Dark Energy: a Challenge for the 21st Century", arXiv: 1004.1493. 
[12] U. Alam, "Constraining Perturbative Early Dark Energy with Current Observations", Astroph. J. 714 (2010) 1460.

[13] T. Holsclaw, et al., "Nonparametric Dark Energy Reconstruction from Supernova Data", Phys. Rev. Lett. 105 (2010) 241302.

[14] S. Nojiri \& S. D. Odintsov, "Introduction to modified gravity and gravitational alternative for dark energy", Phys.Rept. 505 (2011) 59.

[15] U. Alam, Z. Lukic \& S. Bhattacharya, "Galaxy Clusters as a probe of early dark energy", Astroph. J. 727 (2011) 87..

[16] T. Clifton, P. G. Ferreira, A. Padilla \& C. Skordis, "Modified Gravity and Cosmology", Phys.Rept. 513 (2012) 1.

[17] M. J. Mortonson, D, H, Weinberg, \& M. White, "Dark Energy: A Short Review", arXiv: 1401.0046.

[18] V. Sahni \& A. A. Starobinsky, "Reconstructing Dark Energy", Int. J. Mod. Phys. D 15 (2006) 2105.

[19] U. Alam, S. Bag, \& V. Sahni, "Constraining the Cosmology of the Phantom Brane using Distance Measures", Phys. Rev. D 95 (2017) 023524.

[20] P. A. R. Ade, et al., "Planck 2015 results. XIII. Cosmological parameters", Astron. Astrophys. 594 (2016) A13.

[21] P. A. R. Ade, et al., "Planck 2015 results. XIV. Dark energy and modified gravity", Astron. Astrophys. 594 (2016) A14.

[22] V. Sahni, A. Shafieloo \& A. A. Starobinsky, "Model-independent evidence for dark energy evolution from Baryon acoustic oscillations", Astroph. J. 793 (2014) L40.

[23] E. Aubourg, et al., "Cosmological implications of baryon acoustic oscillation (BAO) measurements", Phys. Rev. D 92 (2014) 123516.

[24] T. Delubac, et al., "Baryon Acoustic Oscillations in the Ly forest of BOSS DR11 quasars", Astron. Astrophys. 574 (2015) A59.

[25] S. Alam, et al., "The clustering of galaxies in the completed SDSS-III Baryon Oscillation Spectroscopic Survey: cosmological analysis of the DR12 galaxy sample", arXiv:1607.03155.

[26] D. Scolnic \& R. Kessler, "Measuring Type Ia Supernova Populations of Stretch and Color and Predicting Distance Biases", Astroph. J. Lett., 822 (2016) 35.

[27] S Gupta, \& T.D. Saini, "Direction dependence in supernova data: constraining isotropy", Mon. Not. Roy. Ast. Soc. 407 (2010) 651.

[28] P. S. Corasaniti, "The impact of cosmic dust on supernova cosmology", Mon. Not. Roy. Ast. Soc. 372 (2006) 191.

[29] M. D. Kistler, et al., "The Impact of Metallicity on the Rate of Type Ia Supernovae", Astroph. J. $\mathbf{7 7 0}(2013) 88$.

[30] S. A Uddin, et al., "Cosmological Inference from Host-Selected Type Ia Supernova Samples", arXiv: 1612.07883.

[31] M. V. Pruzhinskaya, E. S. Gorbovskoy \& V. M. Lipunov, "Pure Supernovae and Accelerated Expansion of the Universe", Astron. Lett. 37 (2011) 663.

[32] N. V. Karpenka, F. Feroz \& M. P. Hobson, "Testing the mutual consistency of different supernovae surveys", Mon. Not. Roy. Ast. Soc. 449 (2015) 2405.

[33] J. L. Tonry, et al., "Cosmological Results from High-z Supernovae", Astroph. J. 594 (2003) 1. 
[34] B. J. Barris, et al., "Twenty-Three High-Redshift Supernovae from the Institute for Astronomy Deep Survey: Doubling the Supernova Sample at $z>0.7$ ", Astroph. J. 602 (2004) 571.

[35] A. G. Riess, et al., "Type Ia Supernova Discoveries at $z>1$ From the Hubble Space Telescope: Evidence for Past Deceleration and Constraints on Dark Energy Evolution", Astroph. J. 607 (2004) 665.

[36] P. Astier, et al., "The Supernova Legacy Survey: measurement of $\Omega_{M}, \Omega_{\Lambda}$ and $w$ from the first year data set", Astron. Astrophys. 447(2006) 31.

[37] W. M. Wood-Vasey, et al., "Observational Constraints on the Nature of the Dark Energy: First Cosmological Results from the ESSENCE Supernova Survey", Astroph. J. 666 (2007) 694.

[38] M. Kowalski, et al., "Improved Cosmological Constraints from New, Old and Combined Supernova Datasets", Astroph. J. 686 (2008) 749.

[39] M. Hicken, et al., "CfA3: 185 Type Ia Supernova Light Curves from the CfA", Astroph. J. 700 (2009) 331.

[40] R. Kessler, et al., "First-Year Sloan Digital Sky Survey-II Supernova Results: Hubble Diagram and Cosmological Parameters", Astroph. J. 185 (2009) S32.

[41] N. Suzuki, et al., "The Hubble Space Telescope Cluster Supernova Survey: V. Improving the Dark Energy Constraints Above z⿺辶 1 and Building an Early-Type-Hosted Supernova Sample", Astroph. J. 746 (2012) 85S.

[42] A. Conley, et al., "Supernova Constraints and Systematic Uncertainties from the First 3 Years of the Supernova Legacy Survey", Astroph. J. 192 (2011) S1.

[43] A. Rest, et al., "Cosmological Constraints from Measurements of Type Ia Supernovae discovered during the first 1.5 yr of the Pan-STARRS1 Survey", Astroph. J. 795 (2014) 44.

[44] Betoule, et al., "Improved cosmological constraints from a joint analysis of the SDSS-II and SNLS supernova samples", Astron. Astrophys. 568 (2014) A22.

[45] S. Jha, A. G. Riess, \& R. P. Kirshner, "Improved Distances to Type Ia Supernovae with Multicolor Light-Curve Shapes: MLCS2k2", Astroph. J. 659 (2007) 122.

[46] G. Goldhaber, et al., "Timescale stretch parameterization of Type Ia supernova B-band light curves", Astroph. J. 558 (2001) 359.

[47] J. Guy, et al., "SALT2: using distant supernovae to improve the use of type Ia supernovae as distance indicators", Astron. Astrophys. 466 (2007) 11.

[48] A. Conley, et al., "SiFTO: An Empirical Method for Fitting SN Ia Light Curves", Astroph. J. 681 (2008) 482.

[49] K. S. Mandel, W. M. Wood-Vasey, A. S. Friedman, \& R. P. Kirshner, "Type Ia Supernova Light Curve Inference: Hierarchical Bayesian Analysis in the Near Infrared", Astroph. J. 704 (2009) 629.

[50] K. S. Mandel, G. Narayan, \& R. P. Kirshner, "Type Ia Supernova Light Curve Inference: Hierarchical Models in the Optical and Near-Infrared", Astroph. J. 731 (2011) 120.

[51] M. Sullivan, et al., "SNLS3: Constraints on Dark Energy Combining the Supernova Legacy Survey Three-year Data with Other Probes", Astroph. J. 737 (2011) 102.

[52] J. Johansson, et al., "SNe Ia host galaxy properties from Sloan Digital Sky Survey-II spectroscopy", Mon. Not. Roy. Ast. Soc. 435 (2013) 1680.

[53] M. Hamuy, et al., "The Hubble Diagram of the Calan/Tololo Type IA Supernovae and the Value of $H_{0}$ ", Astron. J. 112 (1996) 2398.

[54] K. Krisciunas, et al., "Hubble Space Telescope Observations of Nine High-Redshift ESSENCE Supernovae", Astron. J. 130 (2005) 2453. 
[55] A. G. Riess, et al., "Cepheid Calibrations Of Modern Type Ia Supernovae: Implications For The Hubble Constant", Astron. J. 117 (1999) 707.

[56] S. Jha, "UBVRI Light Curves of 44 Type Ia Supernovae", et al., Astron. J. 131 (2006) 527.

[57] C. Contreras, et al., "The Carnegie Supernova Project: First Photometry Data Release of Low-Redshift Type Ia Supernovae", Astron. J. 139 (2010) 519.

[58] J. A. Holtzman, et al., "The Sloan Digital Sky Survey-II: Photometry and Supernova IA Light Curves from the 2005 Data", Astron. J. 136 (2008) 2306.

[59] B. P. Schmidt, et al., "The High-z Supernova Search: Measuring Cosmic Deceleration and Global Curvature of the Universe Using Type IA Supernovae", Astroph. J. 507 (1998) 46.

[60] R. Amanullah, et al., "Light curves of five type Ia supernovae at intermediate redshift", Astron. Astrophys. 486 (2008) 375.

[61] R. A. Knop, et al., "New Constraints on $\Omega_{M}, \Omega_{\Lambda}$, and $w$ from an Independent Set of 11 High-Redshift Supernovae Observed with the Hubble Space Telescope", Astroph. J. 598 (2003) 102.

[62] G. Miknaitis, "The ESSENCE Supernova Survey: Survey Optimization, Observations, and Supernova Photometry", et al., Astroph. J. 666 (2007) 674.

[63] Riess et al., "New Hubble Space Telescope Discoveries of Type Ia Supernovae at $z>1$ : Narrowing Constraints on the Early Behavior of Dark Energy", Astroph. J. 659 (2007) 98.

[64] R. Amanullah, et al., "Spectra and Hubble Space Telescope Light Curves of Six Type Ia Supernovae at $0.511<z<1.12$ and the Union2 Compilation", Astroph. J. 716 (2010) 712.

[65] M. Sako, et al., "The Sloan Digital Sky Survey-II Supernova Survey: Search Algorithm and Follow-up Observations", Astron. J. 135 (2008) 348.

[66] M. Hicken, et al., "CfA4: Light Curves for 94 Type Ia Supernovae", Astroph. J. 200 (2012) S12.

[67] R Core Team (2016), "R: A language and environment for statistical computing", $\mathrm{R}$ Foundation for Statistical Computing, Vienna, Austria, URL: https://www.R-project.org/.

[68] J. Lasue, T. Stepinski, \& S.W. Bell, "Automated classification of interplanetary dust particles: Johnson Space Center Cosmic Dust Catalog Volume 15", Meteorit. \& Planet. Sci. 45 (2010) 783.

[69] J. Lasue, et al., "Nonlinear mapping technique for data visualization and clustering assessment of LIBS data: application to ChemCam data", Anal. Bioanal. Chem. 400 (2011) 3247.

[70] I. T. Jolliffe, "Principal Components Analysis", 2nd ed. Springer Verlag, New York, (2002).

[71] M. Davidian \& P. D. Haaland, "Regression and calibration with nonconstant error variance", Chemometrics and Intelligent Laboratory Systems, 9(3), 231 (1990).

[72] D. M. Bates \& D. G. Watts, "Nonlinear Regression Analysis and Its Applications", Wiley, (1988).

[73] D. M. Scolnic, et al., "Color Dispersion and Milky-Way-Like Reddening Among Type Ia Supernovae", Astroph. J. 780 (2013) 37.

[74] R. C. Wolf, et al., "SDSS-II Supernova Survey: An Analysis of the Largest Sample of Type Ia Supernovae and Correlations with Host-Galaxy Spectral Properties", Astroph. J. 821 (2016) 115 . 